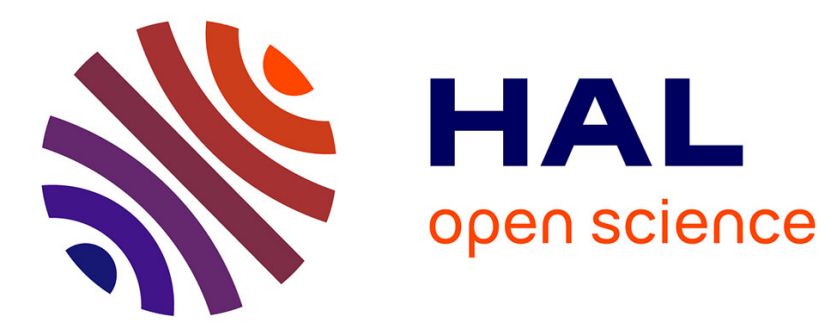

\title{
The rank correlated SLW model of gas radiation in non-uniform media
}

Vladimir Solovjov, Frédéric André, Denis Lemonnier, Brent Webb

\section{To cite this version:}

Vladimir Solovjov, Frédéric André, Denis Lemonnier, Brent Webb. The rank correlated SLW model of gas radiation in non-uniform media. Journal of Quantitative Spectroscopy and Radiative Transfer, 2017, 197, pp.26-44. 10.1016/j.jqsrt.2017.01.034 . hal-02064928

\section{HAL Id: hal-02064928 \\ https://hal.science/hal-02064928}

Submitted on 23 Apr 2019

HAL is a multi-disciplinary open access archive for the deposit and dissemination of scientific research documents, whether they are published or not. The documents may come from teaching and research institutions in France or abroad, or from public or private research centers.
L'archive ouverte pluridisciplinaire HAL, est destinée au dépôt et à la diffusion de documents scientifiques de niveau recherche, publiés ou non, émanant des établissements d'enseignement et de recherche français ou étrangers, des laboratoires publics ou privés. 


\title{
The Rank Correlated SLW Model of Gas Radiation in Non-Uniform Media
}

\author{
Vladimir P. Solovjov ${ }^{1}$, Frederic Andre ${ }^{2}$, Denis Lemonnier ${ }^{3}$ and Brent W. Webb ${ }^{1}$ \\ ${ }^{1}$ Brigham Young University, CTB-435, Provo, UT 84602 USA \\ ${ }^{2}$ Centre de Thermique et d'Energétique de Lyon, INSA de Lyon - 9 rue de la Physique - 69621 Villeurbanne, France \\ ${ }^{3}$ ISAE-ENSMA, BP 40109, 86961 Futuroscope Chasseneuil Cedex, France
}

\begin{abstract}
A comprehensive theoretical development of possible reference approaches in modelling of radiation transfer in non-uniform gaseous media is developed within the framework of the Generalized SLW Model. The notion of absorption spectrum "correlation" adopted currently for global methods in gas radiation is critically revisited and replaced by a less restrictive concept of rank correlated spectrum. Within this framework it is shown that eight different reference approaches are possible, of which only three have been reported in the literature. Among the approaches presented is a novel Rank Correlated SLW Model, which is distinguished by the fact that $i$ ) it does not require the specification of a reference gas thermodynamic state, and ii) it preserves the emission term in the spectrally integrated Radiative Transfer Equation. Construction of this reference model requires only two absorption line blackbody distribution functions, and subdivision into gray gases can be performed using standard quadratures. Consequently, this new reference approach appears to have significant advantages over all other methods, and is, in general, a significant improvement in the global modelling of gas radiation. All reference approaches are summarized in the present work, and their use in radiative transfer prediction is demonstrated for simple example cases. Further, a detailed rigorous theoretical development of the improved methods is provided.
\end{abstract}

\section{Keywords}

Gas radiation, rank correlation of gas absorption spectra, Generalized SLW Method, SLW reference approach, Rank Correlated SLW model 


\section{INTRODUCTION}

Global methods in modelling of radiation transfer in high-temperature gases such as SLW (Spectral Line Weighted-sum-of-gray-gases), ADF (Absorption Distribution Function), FSK (Full Spectrum $k$-distribution), and MBWSGG (Moment-Based Weighted-Sum-of-Gray-Gases) methods, and the hybrid forms of the SLW model with other spectral models [1-12] are computationally the most efficient methods in modelling of gas radiation. These methods can provide high accuracy of prediction of radiation transfer, often comparable to that of the benchmark line-by-line (LBL) method but at significantly lower computational cost. Despite the seeming difference between SLW, ADF, and FSK methods, they all are based on the same fundamental principle in modelling of the gas absorption spectrum, and it has been shown that all of them can be described as particular cases of the recently published Generalized SLW Model [6]. The primary challenge of global methods is to extend the spectral modeling from uniform (isothermal, homogeneous) to nonuniform (non-isothermal, nonhomogeneous) media, for which some additional assumptions regarding the gas absorption spectrum (e.g., assumption of correlated or scaled spectra) must be made. The existing approaches are based on assumptions that can yield large errors in the case of high spatial temperature gradients in the medium. Simply put, the reference approach to handling nonisothermal, nonhomogeneous media involves defining a reference state and correcting all local states relative to that reference state. The main drawback of the reference approach is the absence of a consistent recommendation for the choice of the gas reference state. Despite the interest in the reference approach in global methods, not all possible variations of the method have been discovered. The Generalized SLW Model provides a backdrop to reveal more possibilities for new versions of the reference approach.

The objective of the present paper is to develop and present all possible versions of the SLW reference approach, and investigate and compare their characteristics. The main result is the new Rank Correlated SLW Model which distinguishes itself from other approaches in that it is the only method $i$ ) which does not require the specification of a gas reference state, and $i i$ ) which preserves the emission term in the spectrally integrated Radiative Transfer Equation. This method is introduced in Section 3.5.I to follow, and its detailed outline is presented in Section 3.7. Rigorous theoretical justification of properties for the Rank Correlated SLW model is given in Appendices 1 3. It can be shown that the development presented for the Rank Correlated SLW model in nonuniform media is based on the more general co-monotonic spectral model of gas radiation in nonuniform media employing an arbitrary probability distribution, and is a particular case of its application.

\section{THE SLW METHOD}

\subsection{Spectral Radiative Transfer Equation}

Propagation of radiation in absorbing and emitting gaseous media along a pathlength $s$ in a direction

$\boldsymbol{\Omega}$ is characterized by the spectral radiation intensity $I_{\eta}(s, \boldsymbol{\Omega})$, which is governed by the Radiative Transfer Equation (RTE)

$$
\frac{\partial I_{\eta}(s, \mathbf{\Omega})}{\partial s}=-\kappa_{\eta}(s) I_{\eta}(s, \mathbf{\Omega})+\kappa_{\eta}(s) I_{b \eta}[T(s)]
$$

where $\eta$ is the wavenumber, $I_{b \eta}[T(s)]$ is the spectral Planck blackbody intensity at the local gas temperature $T(s)$, and the local spectral absorption coefficient is defined as 
$\kappa_{\eta}(s)=N(s) Y(s) C_{\eta}(\phi(s)),[1 / m]$ where $C_{\eta}(\phi(s)),\left[\mathrm{m}^{2} / \mathrm{mol}\right]$ is the gas absorption crosssection, and $\phi(s)=\{T(s), Y(s), p(s)\}$ is a symbolic vector notation for the definition of the local gas thermodynamic state at temperature $T(s)$, total pressure $p(s)$, and mole fraction $Y(s)$. High resolution spectral databases such as HITRAN and HITEMP are used to compile the spectral gas absorption cross-section $C_{\eta}(\phi)$.

The Line-by-Line (LBL) method solves the spectral RTE for each wavenumber $\eta$, following which the total intensity of radiation may be obtained by spectral integration over all wavenumbers. The narrow band approximation requires solution of the RTE at hundreds of thousands of spectral points $\eta_{i}$ in the numerical integration:

$$
I(s, \mathbf{\Omega})=\int_{0}^{\infty} I_{\eta}(s, \mathbf{\Omega}) d \eta \approx \sum_{i} I_{\eta_{i}}(s, \mathbf{\Omega}) \Delta \eta_{i}
$$

The SLW model is a particular case of the so-called global family of methods in modeling gas radiation which, in contrast to the Line-by-Line method, starts first with spectral integration of the RTE, and then solves it directly for the spectrally integrated intensity $I(s, \boldsymbol{\Omega})$ for a finite number of discrete values of the absorption cross-section (rather than wavenumber). To determine total radiative properties it may be said that the SLW method integrates over absorption cross-section rather than wavenumber.

\subsection{Characterization of the gas absorption cross-section - ALBDF and inverse ALBDF}

A brief tutorial on the Generalized SLW model is presented here to provide context for the reference approaches identified in sections to follow. The spectral integration of the RTE in the SLW model is made efficient by application of the direct and inverse Absorption Line Blackbody Distribution Function (ALBDF).

The ALBDF $F\left(C, \phi_{g}, T_{b}\right)$ of the absorption cross-section $C$ at a given gas thermodynamic state $\phi_{g}=\{T, Y, p\}$ describes the fraction of the total blackbody radiation power $E_{b}\left(T_{b}\right)=\sigma T_{b}^{4}$ emitted at temperature $T_{b}$ that lies in the part of the spectrum where the gas absorption cross-section $C_{\eta}\left(\phi_{g}\right)$ is below the prescribed value $C$ :

$$
F\left(C, \phi_{g}, T_{b}\right)=\frac{1}{E_{b}\left(T_{b}\right)} \int_{\left\{\eta: C_{\eta}\left(\phi_{g}\right)<C\right\}} E_{b \eta}\left(T_{b}\right) d \eta=\int_{0}^{\infty} H\left(C_{\eta}(\phi)-C\right) \frac{E_{b \eta}\left(T_{b}\right)}{E_{b}\left(T_{b}\right)} d \eta
$$

Here, $H$ is the Heaviside unit-step function. The ALBDF is a strictly increasing function varying between 0 and 1 with respect to the variable $C$, and therefore, it is invertible. The inverse ALBDF $C\left(F, T_{g}, T_{b}\right)$ of the variable $F$ is defined such that

$$
C\left[F\left(C, \phi_{g}, T_{b}\right), \phi_{g}, T_{b}\right]=C \text {, and } F\left[C\left(F, \phi_{g}, T_{b}\right), \phi_{g}, T_{b}\right]=F
$$

The inverse ALBDF can be interpreted as a reordered gas absorption cross-section. The ALBDF is calculated in advance from the high-resolution gas absorption cross-section, and has recently been presented in tabulated form or mathematical correlations $[5,6]$ using the most up-to-date spectral database, HITEMP 2010. The values of the inverse ALBDF may be obtained by inversion of the ALBDF tabulated data, or by polynomial interpolation of mathematical correlations with the help of the method of moments [6]. 


\subsection{The SLW Method - spectral integration of RTE in uniform media}

Consider a uniform gaseous medium at a specified thermodynamic state $\phi_{g}=\{T, Y, p\}$. The spectral gas absorption coefficient at this state is given as $\kappa_{\eta}(\phi)=N Y C_{\eta}(\phi)$, where $C_{\eta}(\phi)$ is the gas spectral absorption cross-section.

\section{SLW histogram spectrum}

As shown in Fig. 1, the continuous line absorption coefficient $C_{\eta}(\phi)$ is modelled in the SLW method by a histogram spectrum in the following way. First, define a set of supplemental absorption cross-sections $\tilde{C}_{j}^{r e f}$ spaced between chosen limiting values $C_{\min }$ and $C_{\max }$ which effectively span the entire range of gas absorption spectrum. As an example, logarithmic spacing has been previously used as $\tilde{C}_{j}^{r e f}=C_{\min }\left(C_{\max } / C_{\min }\right)^{j / n}, j=0,1, \ldots, n$, where $n$ is the number of gray gases in the model. The gray gas absorption cross-sections $C_{j}$ can be chosen arbitrarily in the interval $\tilde{C}_{j-l}<C_{j}<\tilde{C}_{j}$, for example, as a geometric mean $C_{j}=\sqrt{\tilde{C}_{j-1} \tilde{C}_{j}}$. Then the gray gas absorption coefficients are $\kappa_{j}=N Y C_{j}$, and the clear gas is defined as $\kappa_{0}=0$. The supplemental cross-sections define the gray gas and the clear gas spectral intervals:

$$
\Delta_{j}=\left\{\eta: \tilde{C}_{j-1}<C_{\eta}(\phi)<\tilde{C}_{j}\right\}, \Delta_{0}=\left\{\eta: C_{\eta}(\phi)<\tilde{C}_{0}\right\}
$$

Then the SLW histogram spectrum is defined as

$\kappa_{\eta}(\phi)=\kappa_{j}, \eta \in \Delta_{j}, j=0,1, \ldots, n$

\section{Spectral integration of the RTE}

Integration of the spectral RTE over the gray gas spectral intervals $\Delta_{j}, j=0,1, \ldots, n$ yields the following expression:

$\int_{0}^{\infty} \frac{\partial I_{\eta}(s, \boldsymbol{\Omega})}{\partial s} d \eta=-\int_{0}^{\infty} \kappa_{\eta}(s) I_{\eta}(s, \mathbf{\Omega}) d \eta+\int_{0}^{\infty} \kappa_{\eta}(s) I_{b \eta}[T(s)] d \eta$

Because the spectral gray gas intervals are fixed for all spatial locations in a uniform medium, the order of differentiation and integration on the left-hand side of the equation can be interchanged. That yields the gray gas RTEs:

$$
\frac{\partial I_{j}(s, \mathbf{\Omega})}{\partial s}=-\kappa_{j} I_{j}(s, \mathbf{\Omega})+a_{j} \kappa_{j} I_{b \eta}(T)
$$

where

$$
I_{j}(s, \mathbf{\Omega})=\int_{\Delta_{j}} I_{\eta}(s, \mathbf{\Omega}) d \eta
$$

is defined as the gray gas intensity, and

$$
a_{j}=F\left(\tilde{C}_{j}, \phi_{g}=\phi, T_{b}=T\right)-F\left(\tilde{C}_{j-1}, \phi_{g}=\phi, T_{b}=T\right)
$$

are the gray gas weights. After solution of the gray gas RTEs, the total intensity can be found by a summation over all gray gases: 


$$
I(s, \mathbf{\Omega})=\sum_{j=0}^{n} \int_{\Delta_{j}} I_{\eta}(s, \mathbf{\Omega}) d \eta=\sum_{j=0}^{n} I_{j}(s, \mathbf{\Omega})
$$

\subsection{The SLW Method - spectral integration of RTE in non-uniform media}

In non-uniform (nonisothermal/nonhomogeneous) media, the gas absorption cross-section $C_{\eta}(\phi)$ varies with spatial location $s$ if the thermodynamic state $\phi(s)$ depends on location. In this case the intersection of the fixed supplemental absorption cross-sections $\tilde{C}_{j}$ with the absorption crosssections $\phi_{1}=\phi\left(s_{1}\right)$ and $\phi_{2}=\phi\left(s_{2}\right)$ at different spatial locations $s_{1}$ and $s_{2}$ produces different gray gas wavenumber intervals, $\Delta_{j}\left(s_{l}\right) \neq \Delta_{j}\left(s_{2}\right)$. Therefore, in the spectral integration of the RTE, interchange of differentiation with respect to the spatial variable $s$ and integration with respect to the wavenumber $\eta$ over the gray gas intervals $\Delta_{j}(s)=\left\{\left[a_{i, j}(s), b_{i, j}(s)\right]\right\}$ will produce the so-called Leibnitz terms [2] due to variation of the limits of integration:

$$
\begin{aligned}
\int_{\Delta_{j}(s)} \frac{\partial}{\partial s} I_{\eta}(s, \boldsymbol{\Omega}) d \eta=\frac{\partial}{\partial s} \int_{\Delta_{j}(s)} I_{\eta}(s, \boldsymbol{\Omega}) d \eta \\
+\overbrace{\sum_{i}\left\{I_{\eta}\left[s, \eta=b_{i, j}(s), \boldsymbol{\Omega}\right] \frac{d b_{i, j}(s)}{d s}-I_{\eta}\left[s, \eta=a_{i, j}(s), \boldsymbol{\Omega}\right] \frac{d a_{i, j}(s)}{d s}\right\}}^{\text {Leibnitz terms }}
\end{aligned}
$$

It is impossible to account for these numerous Leibnitz terms, but their neglect can yield a significant error in prediction of radiation transfer in gas media with high non-uniformity. To eliminate Leibnitz terms from the spectrally integrated RTE, the gray gas intervals must be fixed for all spatial locations, $\Delta_{j}(s)=\Delta_{j}=$ const :

$$
\int_{\Delta_{j}} \frac{\partial}{\partial s} I_{\eta}(s, \mathbf{\Omega}) d \eta=\frac{\partial}{\partial s} \int_{\Delta_{j}} I_{\eta}(s, \mathbf{\Omega}) d \eta+\overline{\text { Leitritexms }}
$$

The gray gas intervals may be fixed by choosing different supplemental absorption cross-sections $\tilde{C}_{j}(s)$ at different locations in such a way that they define the same spectral intervals $\Delta_{j}=$ const for all locations, as illustrated in Fig. 2. In the SLW model, this is ensured by invoking the reference approach.

\subsection{The SLW reference approach - "ideal spectrum" assumption}

The SLW reference approach is based on the assumption of "ideal behavior" of the gas absorption cross-section in non-uniform media defined as follows. For an arbitrary prescribed reference value $C^{r e f}$ of the absorption cross-section $C_{\eta}\left(\phi_{\text {ref }}\right)$ at the chosen reference state $\phi_{\text {ref }}$, there exists a value $C^{l o c}$ such that the line $C=C^{r e f}$ intersects the absorption cross-section $C_{\eta}\left(\phi_{\text {ref }}\right)$ at the same wavenumbers $\eta$ at which the line $C=C^{\text {loc }}$ intersects the absorption cross-section $C_{\eta}\left(\phi_{l o c}\right)$ at any local thermodynamic state. The implication of this observation is that the spectral intervals $\Delta=\left\{\eta: C_{\eta}\left(\phi_{r e f}\right)<C^{r e f}\right\}=\left\{\eta: C_{\eta}\left(\phi_{l o c}\right)<C^{l o c}\right\}$ are equal and therefore, the values of the ALBDF at $C^{\text {loc }}$ and $C^{\text {ref }}$ calculated by integration over this interval with the same blackbody source temperature $T_{b}$ will be the same as illustrated in Fig. 3:

$$
F\left(C^{l o c}, \phi_{l o c}, T_{r e f}\right)=F\left(C^{r e f}, \phi_{r e f}, T_{r e f}\right)
$$


This implicit equation is used in the SLW reference approach to find the local supplemental absorption cross-sections $\tilde{C}_{j}^{l o c}$ which maintain the same spectral intervals, and, therefore, eliminate the Leibnitz terms in the gray gas RTEs. An iterative technique, such as bisection or NewtonRaphson methods, has been used for solution of this implicit equation.

It should be noted that this global method approach is similar to the correlated $k$-distribution method used for narrow bands in non-uniform media [13]. The "ideal behavior" assumption for spectra is valid for the case of scaled spectra when the absorption cross-section can be expressed as a simple product $C_{\eta}(\phi)=f(\phi) h(\eta)$, separating the dependence on thermodynamic state $\phi$ and wavenumber $\eta$ (Milne- Eddington approximation).

\section{Outline of the SLW Reference Approach [2]}

The SLW reference approach thus follows the steps below:

1. Choose a reference state $\phi_{r e f}=\left\{T_{r e f}, Y_{r e f}, p_{r e f}\right\}$ by some appropriate weighting of the properties over the domain (e.g., simple volumetric average).

2. Choose a set of reference supplemental cross-sections $\tilde{C}_{j}^{\text {ref }}$ (log-spaced or optimized).

3. Find the corresponding local supplemental cross-sections $\tilde{C}_{j}^{\text {loc }}$ by solving the implicit equations:

$$
F\left(\tilde{C}_{j}^{l o c}, \phi_{l o c}, T_{r e f}\right)=F\left(\tilde{C}_{j}^{r e f}, \phi_{r e f}, T_{r e f}\right)
$$

4. Calculate the local gray gas absorption coefficients from the supplemental cross-sections:

$$
\kappa_{j}(s)=N_{l o c} Y_{l o c} C_{j}^{l o c}=N(s) Y(s) \sqrt{\tilde{C}_{j}^{l o c} \tilde{C}_{j-1}^{l o c}}
$$

5. Calculate the local weights of the gray gas absorption coefficients:

$$
a_{j}(s)=a_{j}^{l o c}=F\left(\tilde{C}_{j}^{r e f}, \phi_{r e f}, T_{b}=T_{l o c}\right)-F\left(\tilde{C}_{j-1}^{r e f}, \phi_{r e f}, T_{b}=T_{l o c}\right)
$$

6. Solve the gray gas RTE in the non-isothermal, non-homogeneous medium using the gas property parameters found in steps 1-5:

$$
\frac{\partial I_{j}(s)}{\partial s}=-\kappa_{j}(s) I_{j}(s)+\kappa_{j}(s) a_{j}(s) I_{b}[T(s)]
$$

The ADF reference approach [8] differs from the original SLW reference approach by the way in which the gray gas weights are calculated. Instead of calculating the weights at the reference supplemental cross-sections $\tilde{C}_{j}^{\text {ref }}$, the ADF method calculates the weights with the help of the previously determined local supplemental cross-sections.

$$
a_{j}(s)=a_{j}^{l o c}=F\left(\tilde{C}_{j}^{l o c}, \phi_{l o c}, T_{b}=T_{l o c}\right)-F\left(\tilde{C}_{j-1}^{l o c}, \phi_{l o c}, T_{b}=T_{l o c}\right)
$$

The two methods differ in their ability to handle exactly the transmissivity and emissivity of a gas layer as is discussed in [8].

\section{THE RANK CORRELATED SLW MODEL}

\subsection{Concept of "correlated" gas absorption spectrum currently adopted in global methods}

The concept of "ideal spectrum" discussed in [2] was reformulated in [9, p. 660] to a concept of "correlated" spectrum as the relationship between absorption coefficient $\kappa_{\eta}(\eta, \phi)$ at an arbitrary state $\phi$ and the absorption coefficient $\kappa_{\eta}(\eta)=\kappa_{\eta}\left(\eta, \phi_{0}\right)$ at some chosen reference state $\phi_{0}$ in the following way: At every wavenumber $\eta$ where the reference absorption spectrum $\kappa_{\eta}\left(\eta, \phi_{0}\right)$ has 
one and the same value $k$, the local absorption spectrum $\kappa_{\eta}(\eta, \phi)$ always also has one unique value $k^{*}(\phi, k)$. This may be expressed mathematically as [9]

$\kappa_{\eta}(\eta, \phi)=k_{\eta}^{*}\left(\phi, k_{\eta}\right)$

If the ratio $k_{\eta}^{*}\left(\phi, k_{\eta}\right) / k_{\eta}$ is constant, then the absorption coefficient is said to be scaled, and the ratio $u\left(\phi, \phi_{0}\right)=k_{\eta}^{*}\left(\phi, k_{\eta}\right) / k_{\eta}$ is called the scaling function, which depends only on the local and reference states. If this ratio depends on the reference absorption coefficient $\kappa_{\eta}\left(\eta, \phi_{0}\right)$, then the spectra are said to be correlated. These definitions are summarized in [9] as

$$
\kappa_{\eta}(\eta, \phi)=\left\{\begin{array}{cc}
k_{\eta}^{*}\left(\phi, k_{\eta}\right) & \text { correlated } \\
u\left(\phi, \phi_{0}\right) k_{\eta}(\eta) & \text { scaled }
\end{array}\right.
$$

It is suggested here that the definition given of the assumption of correlated spectrum is, in fact, not really an assumption. Due to the presence of $\eta$ in $\kappa_{\eta}(\eta, \phi)$ the relationship can be established point by point with respect to wavenumber $\eta$ for any arbitrary absorption spectrum regardless of whether the spectrum is related or not. The concept of spectral "correlation" presented in [9] limits the possibility of further improvement of global modelling of gas radiation in non-uniform medium.

In this work the term "correlated spectrum" will be understood in the sense of "ideal spectrum" as defined in [2]. It is important to note that all existing definitions of spectral correlation are essentially based on a requirement to specify the reference absorption spectrum $\kappa_{\eta}\left(\eta, \phi_{0}\right)$ at some chosen gas thermodynamic state $\phi_{0}$. All existing reference approaches of the SLW, ADF, and FSK methods involve the gas absorption coefficient at the reference state as their starting point.

\subsection{The rank correlated gas absorption spectrum}

Further development of the SLW model in non-uniform media can be achieved by modification of the assumption of "ideal spectrum" or "correlated spectrum" discussed above to a less restrictive assumption of rank correlation regarding the relationship between absorption spectra at different thermodynamic states. The assumption of the rank correlated spectrum is defined here as follows: Let $\phi_{1}$ and $\phi_{2}$ represent two arbitrary gas thermodynamic states, and let $\Delta_{l}=\left\{\eta: C_{\eta}\left(\phi_{l}\right)<C_{\eta^{\prime}}\left(\phi_{l}\right)\right\}$ and $\Delta_{2}=\left\{\eta: C_{\eta}\left(\phi_{2}\right)<C_{\eta^{\prime}}\left(\phi_{2}\right)\right\}$ be the wavenumber intervals defined by the values of absorption cross-sections $C_{\eta^{\prime}}\left(\phi_{1}\right)$ and $C_{\eta^{\prime}}\left(\phi_{2}\right)$ at the same arbitrary value of wavenumber $\eta^{\prime}$. If the intervals $\Delta_{l}=\Delta_{2}$ are identical, the spectra $C_{\eta}\left(\phi_{1}\right)$ and $C_{\eta}\left(\phi_{2}\right)$ (neither of which is designed as a reference spectrum) are said to be rank correlated. This definition is visualized in Fig. 4. Rank correlation establishes the relationship between the gas absorption cross-sections at two different gas thermodynamic states, and it does not require specification of any reference thermodynamic state $\phi_{\text {ref }}$ or reference absorption cross-section $C_{\eta}^{r e f}=C_{\eta}\left(\phi_{r e f}\right)$.

Consider the inverse ALBDF $C\left(F, \phi, T_{b}\right)$ at different thermodynamic states $\phi$ for the same fixed source blackbody source temperature $T_{b}$. According to the assumption of rank correlated spectrum, for a fixed value of the $F$-variable and fixed blackbody source temperature $T_{b}$, the wavenumber intervals are defined as follows

$$
\left\{\eta: C_{\eta}\left(\phi_{1}\right)<C\left(F, \phi_{1}, T_{b}\right)\right\}=\left\{\eta: C_{\eta}\left(\phi_{2}\right)<C\left(F, \phi_{2}, T_{b}\right)\right\}=\Delta
$$

These wavenumber intervals are identical. The rigorous proof of this property of rank correlated spectrum is given in Appendix 1. Because of this property, the inverse $\operatorname{ALBDF} C\left(F, \phi_{m}, T_{b}\right)$, 
$m=1,2, \ldots$ can be defined as the rank correlated or co-monotonic reordered continuous absorption cross-sections as depicted in Fig. 5.

The thermodynamic states $\phi_{m}$ can be any local thermodynamic states in the medium. It is important to note that no reference thermodynamic state is involved in this definition of rank correlated reordered absorption cross-sections. Thus, in the SLW model, this approach will yield piecewise constant rank correlated reordered gray gas absorption cross-sections, expressed as $C\left(F_{j}, \phi_{m}, T_{b}\right)\left[H\left(F-\tilde{F}_{j-l}\right)-H\left(F-\tilde{F}_{j}\right)\right]$, where $H(F)$ is the Heaviside unit-step function. The rank correlated reordered absorption cross-sections are shown schematically in Fig. 6.

As stated previously, the concept of rank correlated reordered absorption cross-section $C\left(F, \phi_{m}, T_{b}\right)$ considered in this work is a particular case of a more general approach to co-monotonic global spectral modelling of gas radiation in non-uniform media based on arbitrary probability measures, where it can be shown that if two arbitrary constants $k_{1}, k_{2}>0$, one of two conditions exist:

$$
\left\{\eta: \kappa_{\eta}\left(\phi_{1}\right)<k_{1}\right\} \subseteq\left\{\eta: \kappa_{\eta}\left(\phi_{2}\right)<k_{2}\right\} \text { or }\left\{\eta: \kappa_{\eta}\left(\phi_{1}\right)<k_{1}\right\} \supseteq\left\{\eta: \kappa_{\eta}\left(\phi_{2}\right)<k_{2}\right\}
$$

Consequently, the corresponding spectral absorption coefficients $\kappa_{\eta}\left(\phi_{1}\right)$ and $\kappa_{\eta}\left(\phi_{2}\right)$ are comonotonic (for common monotonicity).

An equivalent relationship in terms of absorption cross-sections and the ALBDF can be established for SLW modelling. In Appendix 3, it is shown that if gas spectra at two different thermodynamic states $C_{\eta}\left(\phi_{1}\right)$ and $C_{\eta}\left(\phi_{2}\right)$ are rank correlated, then for any two prescribed values $C_{1}$ and $C_{2}$, either the set of all wavenumbers $\eta$ such that $C_{\eta}\left(\phi_{1}\right)<C_{1}$ is a subset of all wavenumbers $\eta$ for which $C_{\eta}\left(\phi_{2}\right)<C_{2}$, or vice versa. Mathematically, this may be stated

$$
\left\{\eta: C_{\eta}\left(\phi_{1}\right)<C_{1}\right\} \subseteq\left\{\eta: C_{\eta}\left(\phi_{2}\right)<C_{2}\right\} \text { or }\left\{\eta: C_{\eta}\left(\phi_{1}\right)<C_{1}\right\} \supseteq\left\{\eta: C_{\eta}\left(\phi_{2}\right)<C_{2}\right\} \text {. }
$$

For example, if one assumes that the first inclusion $\left\{\eta: C_{\eta}\left(\phi_{1}\right)<C_{1}\right\} \subseteq\left\{\eta: C_{\eta}\left(\phi_{2}\right)<C_{2}\right\}$ is true, then the relationship between the ALBDFs at two states in SLW terms yields:

$$
\begin{aligned}
F\left(C_{1}, \phi_{1}, T_{b}\right) & =\int_{0}^{\infty} H\left(C_{\eta}\left(\phi_{1}\right)-C_{1}\right) \frac{I_{b \eta}\left(T_{b}\right)}{I_{b}\left(T_{b}\right)} d \eta=\int_{\left\{\eta: C_{\eta}\left(\phi_{1}\right)<C_{1}\right\}} \frac{I_{b \eta}\left(T_{b}\right)}{I_{b}\left(T_{b}\right)} d \eta \\
& \leq \int_{\left\{\eta: C_{\eta}\left(\phi_{2}\right)<C_{2}\right\}} \frac{I_{b \eta}\left(T_{b}\right)}{I_{b}\left(T_{b}\right)} d \eta \quad=F\left(C_{2}, \phi_{2}, T_{b}\right)
\end{aligned}
$$

Therefore, $F\left(C_{1}, \phi_{1}, T_{b}\right) \leq F\left(C_{2}, \phi_{2}, T_{b}\right)$. Further, in this case,

$$
H\left(C_{\eta}\left(\phi_{1}\right)-C_{1}\right) H\left(C_{\eta}\left(\phi_{2}\right)-C_{2}\right)=H\left(C_{\eta}\left(\phi_{1}\right)-C_{1}\right)
$$

Therefore, the joint distribution function of absorption cross-sections at two different thermodynamic states $\phi_{1}$ and $\phi_{2}$ in this case is equal to the minimum of the two functions $F\left(C_{1}, \phi_{1}, T_{b}\right)$ and $F\left(C_{2}, \phi_{2}, T_{b}\right)$ :

$$
\begin{aligned}
F\left(C_{1}, C_{2} ; \phi_{1}, \phi_{2}, T_{b}\right) & =\int_{0}^{\infty} H\left(C_{\eta}\left(\phi_{1}\right)-C_{1}\right) H\left(C_{\eta}\left(\phi_{2}\right)-C_{2}\right) \frac{I_{b \eta}\left(T_{b}\right)}{I_{b}\left(T_{b}\right)} d \eta \\
& =\int_{0}^{\infty} H\left(C_{\eta}\left(\phi_{1}\right)-C_{1}\right) \frac{I_{b \eta}\left(T_{b}\right)}{I_{b}\left(T_{b}\right)} d \eta
\end{aligned}
$$




$$
=F\left(C_{1}, \phi_{1}, T_{b}\right)=\min \left\{F\left(C_{1}, \phi_{1}, T_{b}\right), F\left(C_{2}, \phi_{2}, T_{b}\right)\right\}
$$

The same result, $F\left(C_{1}, C_{2} ; \phi_{1}, \phi_{2}, T_{b}\right)=\min \left\{F\left(C_{1}, \phi_{1}, T_{b}\right), F\left(C_{2}, \phi_{2}, T_{b}\right)\right\}$, can be obtained if the second condition $\left\{\eta: C_{\eta}\left(\phi_{1}\right)<C_{1}\right\} \supseteq\left\{\eta: C_{\eta}\left(\phi_{2}\right)<C_{2}\right\}$ is true. It can be demonstrated that in this case, the gas spectra at states $\phi_{1}$ and $\phi_{2}$ are related through the equation $F\left(C_{\eta}\left(\phi_{1}\right), \phi_{1}, T_{b}\right)=F\left(C_{\eta}\left(\phi_{2}\right), \phi_{2}, T_{b}\right)$ for any wavenumber $\eta$, and thus, for any fixed value $\xi \in(0,1)$ it may be stated

$\xi=F\left(C\left(\xi, \phi_{1}, T_{b}\right), \phi_{1}, T_{b}\right)=F\left(C\left(\xi, \phi_{2}, T_{b}\right), \phi_{2}, T_{b}\right)$.

This relationship does not require the definition of any reference state because it is valid for any two states, and preservation of the intervals of integration is guaranteed. Therefore, construction of the Rank Correlated SLW model with the help of the inverse ALBDF (reordered absorption coefficient) at any local state is given by the equation

$$
C_{j}\left(\phi_{l o c}\right)=C\left(F_{j}, \phi_{l o c}, T_{b}\right)
$$

without the need of specification of the reference state. This fact will be applied in Section 3.4 for the construction of the Rank Correlated SLW model which starts with subdivision of the $F$-variable into fixed $F_{j}, \tilde{F}_{j}, j=0,1, \ldots, n$ values. The rank correlated gray gas reordered absorption coefficients $C_{j}^{\text {loc }}$ can be found at any local gas thermodynamic state without need of the reference spectrum. This approach preserves ranking of the local gray gas absorption coefficient $C_{j}^{\text {loc }}$ at all local states, and maintains the gray gas spectral intervals of integration $\Delta_{j}$ fixed at all states. The rigorous proof of the preservation of spectral intervals of integration by the Rank Correlated SLW model is given in Appendix 3. This simply means that according to the assumption of rank correlation of the gas spectrum, only a discretization of the interval of variations of the variable $F \in(0,1)$ is required to define 1$)$ the gray gas absorption coefficients in any state and, as a consequence, 2) the spectral intervals over which the gas spectra are assumed to be constant; they are considered to be the same for any state and do not depend on the choice of any reference thermophysical condition.

\subsection{Comments on reference gas thermodynamic state and reference partition into gray gases}

The SLW reference approach is based on introduction of different reference quantities. It is for this reason that it is important to clarify their definition and meaning here.

The gas reference thermodynamic state is denoted as $\phi_{r e f}=\left\{T_{r e f}, Y_{r e f}, p_{r e f}\right\}$ for a single gas. The reference mole fractions of gas species $Y_{m, r e f}$ and the reference soot volume fraction $f_{v}^{\text {ref }}$ should be added in the case of gas mixtures with soot. The reference state defines the reference absorption cross-section $C_{\eta}^{r e f}=C_{\eta}\left(\phi_{r e f}\right)$ and the corresponding reference absorption coefficient.

The ALBDF calculated at the reference conditions is $F\left(C, \phi_{g}=\phi_{r e f}, T_{b}=T_{\text {ref }}\right)$, where the reference blackbody source temperature $T_{b}=T_{\text {ref }}$ is a fixed temperature not necessarily equal to the temperature defining the reference state. This temperature fixes the reference Planck blackbody intensity $I_{b \eta}\left(T_{r e f}\right)$ which defines a reference measure $I_{b \eta}\left(T_{r e f}\right) d \eta$, and, as stated correctly by Modest, is "only a mathematical convenience and its choice does not affect the accuracy of calculations [9, p. 665]." 
Consider the reference partition of the $C$-variable. The set of the reference supplemental crosssections $\tilde{C}_{j}^{r e f}$ defines the reference values of absorption cross-sections $C_{j}^{\text {ref }}=\sqrt{\tilde{C}_{j}^{\text {ref }} \tilde{C}_{j-1}^{\text {ref }}}$ and corresponding partition into gray gas spectral intervals $\Delta_{j}=\left\{\eta: \tilde{C}_{j-I}^{r e f}<C_{\eta}\left(\phi_{r e f}\right)<\tilde{C}_{j}^{\text {ref }}\right\}$. In the reference approach these spectral intervals should be held constant for any spatial location of the gas medium. Partition of the $C$-variable can be done in a variety of ways, but it is usually logarithmically evenly spaced between chosen values of $C_{\min }$ and $C_{\max }$ which effectively cover the entire range of gas absorption spectrum.

Reference partition into gray gases can be performed starting either by partition of the $C$-variable or by partition of the $F$-variable: $i$ ) If partition into $\tilde{C}_{j}^{\text {ref }}$ is chosen then partition into $\tilde{F}_{j}^{\text {ref }}$ is calculated as $\tilde{F}_{j}^{r e f}=F\left(\tilde{C}_{j}^{r e f}, \phi_{g}=\phi_{r e f}, T_{b}=T_{r e f}\right)$ and $F_{j}^{r e f}=F\left(C_{j}^{r e f}, \phi_{g}=\phi_{r e f}, T_{b}=T_{r e f}\right) . \quad$ ii) Alternatively, reference partition of the $F$-variable into arbitrary fixed values $\tilde{F}_{j}^{\text {ref }}$ and $F_{j}^{\text {ref }}$ can be performed without a need of the gas thermodynamic state. Subdivision into gray gases may be then performed with the local absorption cross-sections as $\tilde{C}_{j}^{l o c}=C\left(\tilde{F}_{j}^{r e f}, \phi_{g}=\phi_{l o c}, T_{b}=T_{\text {ref }}\right)$, preserving the same gray gas spectral intervals $\Delta_{j}=\left\{\eta: \tilde{C}_{j-1}^{l o c}<C_{\eta}\left(\phi_{l o c}\right)<\tilde{C}_{j}^{l o c}\right\}$ for any spatial location. As noted previously, the choice of the reference blackbody source temperature $T_{b}=T_{\text {ref }}$ is arbitrary and it is needed only to maintain rank correlation of the reordered absorption cross-sections at different locations. The corresponding partition into reference supplemental cross-sections can be made with the inverse ALBDF as $\tilde{C}_{j}^{r e f}=C\left(\tilde{F}_{j}^{r e f}, \phi_{g}=\phi_{r e f}, T_{b}=T_{r e f}\right)$.

It is important to note the difference between the choice of the gas reference temperature $T_{g}^{r e f}=T_{\text {ref }}$ defining the reference thermodynamic state $\phi_{r e f}=\left\{T_{r e f}, Y_{r e f}, p_{r e f}\right\}$, and the reference blackbody source temperature $T_{b}=T_{r e f}$ in the Planck blackbody intensity $I_{b \eta}\left(T_{b}=T_{r e f}\right)$.

The main drawback of current reference approaches is that predictions can be quite sensitive to the choice of the reference state. There are a number of proposed methods for defining the reference state, including volumetric averaging, Planck emission $\left(T^{4}\right)$ averaging, the maximum temperature of the medium, temperature of an equivalent isothermal medium, a temperature which yields the same emissivity, etc. However, these choices are not universal, have questionable theoretical justification, and they cannot be applied in all cases. Consequently, no systematic recommendation can be given. A very detailed analysis of the factors which influence non-correlatedness of the absorption coefficient of real gases, and of the importance of the choice of the reference state is presented in [9] with a conclusion that the choice of $\phi_{\text {ref }}$ is very important and should be optimized for each unique problem. At the same time it is again noted that the choice of the reference blackbody temperature $T_{b}=T_{\text {ref }}$ used in the Planck function is only a mathematical convenience.

\subsection{Construction of the Rank Correlated Generalized SLW Reference Approach}

The development of possible reference approaches begins with the Generalized SLW Method, starting with subdivision of either $C$ or $F$ variables as follows:

1) The $C$-variable is subdivided into supplemental cross-sections $\tilde{C}_{j}^{r e f}$ spaced between chosen values $C_{\min }$ and $C_{\max }$ which effectively cover the entire range of the gas absorption spectrum. For example, logarithmic spacing has been previously used as $\tilde{C}_{j}^{r e f}=C_{\min }\left(C_{\max } / C_{\min }\right)^{j / n}$, $j=0,1, \ldots, n$, where $n$ is the number of gray gases in the model.

2) Because $F$ varies between 0 and 1 and the subdivision increment of the $F$ variable plays the role of weights of gray gas absorption coefficients in the spectral integration, a reasonable 
subdivision may be made with integral quadratures, the positive abscissa $x_{j}>0$ and corresponding weights $w_{j}, j=1,2, \ldots, N$ of the Gaussian-Legendre quadratures for integration over the interval $[-1,1]$. Consequently, the discretization into values $\tilde{F}_{j}^{\text {ref }}$ which define the gray gas intervals, and into values $F_{j}^{\text {ref }}$ which define the absorption cross-sections, is performed by transformation of the Gaussian quadratures from $[0,1]$ into the interval $\left[F_{\min }, F_{\max }\right]$ :

$\tilde{F}_{0}^{r e f}=F_{\min }, \quad \tilde{F}_{j}^{r e f}=F_{\min }+\left(F_{\max }-F_{\min }\right) \sum_{k=1}^{j} w_{k}, \quad F_{j}^{r e f}=F_{\min }+x_{j} \cdot\left(F_{\max }-F_{\min }\right), \quad j=1,2, \ldots, N$

Generally speaking, the SLW reference approach has the possibility of two gas thermodynamic states $\phi_{\text {ref }}=\left(T_{r e f}, Y_{r e f}, p_{\text {ref }}\right)$ and $\phi_{l o c}=\left(T_{l o c}, Y_{l o c}, p_{l o c}\right)$, two absorption cross-sections $C_{\eta}\left(\phi_{g}=\phi_{\text {ref }}\right)$ and $C_{\eta}\left(\phi_{g}=\phi_{l o c}\right)$, and two values of the blackbody temperature $T_{b}=T_{r e f}, T_{b}=T_{l o c}$. Therefore, in total there are four possible ALBDFs, $F(C)$, and corresponding inverse ALBDFs, $C(F)$ :

$$
\begin{array}{ll}
F\left(C, \phi_{g}=\phi_{r e f}, T_{b}=T_{r e f}\right), & C\left(F, \phi_{g}=\phi_{r e f}, T_{b}=T_{r e f}\right) \\
F\left(C, \phi_{g}=\phi_{l o c}, T_{b}=T_{r e f}\right), & C\left(F, \phi_{g}=\phi_{l o c}, T_{b}=T_{r e f}\right) \\
F\left(C, \phi_{g}=\phi_{r e f}, T_{b}=T_{l o c}\right), & C\left(F, \phi_{g}=\phi_{r e f}, T_{b}=T_{l o c}\right) \\
F\left(C, \phi_{g}=\phi_{l o c}, T_{b}=T_{l o c}\right), & C\left(F, \phi_{g}=\phi_{l o c}, T_{b}=T_{l o c}\right)
\end{array}
$$

Two methods are used to establish dependence between reference and local spectra $C_{\eta}\left(\phi_{g}=\phi_{\text {ref }}\right)$ and $C_{\eta}\left(\phi_{g}=\phi_{l o c}\right)$ :

1) Dependence with respect to reference blackbody source temperature $T_{b}=T_{\text {ref }}$, or

2) Dependence with respect to local blackbody source temperature $T_{b}=T_{l o c}$.

Depending on the choice of the reference discretization into gray gases, the choice of the blackbody temperature, and the choice of the local or reference supplemental cross-sections for calculation of the local gray gas weights, there are thus eight possible versions of the SLW reference approach.

Simply put, the objective of the SLW reference approach is to find:

1) the local supplemental cross-sections $\tilde{C}_{j}^{\text {loc }}$ which define the local gray gas absorption coefficient

$$
\kappa_{j}^{l o c}=N_{l o c} Y_{l o c} \sqrt{\tilde{C}_{j-l}^{l o c} \tilde{C}_{j}^{l o c}}=N_{l o c} Y_{l o c} C_{j}^{l o c}, \text { and }
$$

2) the supplemental values $\tilde{F}_{j}^{\text {loc }}$, which define the local weights of gray gas absorption coefficients $a_{j}^{l o c}=\tilde{F}_{j}^{l o c}-\tilde{F}_{j-1}^{l o c}$.

Figure 7 demonstrates the principles of the reference approach confirming conservation of the spectral intervals of integration at different states. Figure 8 reveals four possible ALBDFs generated using combinations of local and reference states $\left(\phi_{r e f}, \phi_{l o c}, T_{b}=T_{r e f}, T_{b}=T_{l o c}\right)$ as outlined above. These four ALBDFs graphed qualitatively in the four quadrants of the figure are generated with the possible combinations of reference and local variables, and each has a corresponding inverse ALBDF. Figure 2 reflects all steps in the possible reference approaches and outlines the difference between the approaches. Referencing the four quadrants of Fig. 8, classification and notations for the different versions of the SLW reference approach are summarized in the panels of Fig. 9, designated as methods I.1.1 - I.2.2 and II.1.1 - II.2.2.

\subsection{Outline of all possible versions of the SLW reference approach}


The possible versions of the reference approach may be classified generally as $i$ ) those that treat the spectrum relationship for the blackbody source temperature at the reference temperature $T_{b}=T_{\text {ref }}$ (designated Method I.x.x), and ii) those that treat the spectrum relationship for the blackbody source temperature at the local temperature, $T_{b}=T_{\text {loc }}$ (designated Method II.X.x). The steps in treating the spectral relationship are summarized below for all possible approaches.

\subsection{Spectrum relationship at the reference blackbody source temperature $T_{b}=T_{r e f}$}

Method I.1.1 This method was the first published SLW reference approach, presented in [2].

1) Start with subdivision of the $C$-variable into supplemental cross-sections $\tilde{C}_{j}^{r e f}, j=0,1, \ldots, n$.

2) Calculate the reference values $\tilde{F}_{j}^{\text {ref }}$ with the direct ALBDF $\tilde{F}_{j}^{r e f}=F\left(\tilde{C}_{j}^{r e f}, \phi_{r e f}, T_{r e f}\right)$

3) Calculate the local supplemental absorption cross-sections with the inverse ALBDF $\tilde{C}_{j}^{l o c}=C\left(\tilde{F}_{j}^{r e f}, \phi_{l o c}, T_{r e f}\right)$

4) Calculate the local gray gas absorption coefficients $\kappa_{j}^{l o c}=N^{l o c} Y^{l o c} \sqrt{\tilde{C}_{j-1}^{l o c} \tilde{C}_{j}^{l o c}}, \kappa_{0}^{l o c}=0$

5) Local weights are then calculated using the ALBDF at the reference cross-sections $\tilde{C}_{j}^{r e f}$ $a_{j}^{\text {loc }}=F\left(\tilde{C}_{j}^{r e f}, \phi_{r e f}, T_{l o c}\right)-F\left(\tilde{C}_{j-1}^{r e f}, \phi_{r e f}, T_{l o c}\right), a_{0}^{\text {loc }}=F\left(\tilde{C}_{0}^{r e f}, \phi_{r e f}, T_{l o c}\right)$

Method I.1.2 This method is a modification of the SLW reference approach [3] and the ADF method [8]. It differs from the original SLW model (Model I.1.1) only by the manner in which the local gray gas weights are calculated.

1) Start with subdivision of the $C$-variable into supplemental cross-sections $\tilde{C}_{j}^{r e f}, j=0,1, \ldots, n$.

2) Calculate the reference values $\tilde{F}_{j}^{\text {ref }}$ with the direct ALBDF $\tilde{F}_{j}^{r e f}=F\left(\tilde{C}_{j}^{r e f}, \phi_{r e f}, T_{r e f}\right)$

3) Calculate the local supplemental absorption cross-sections with the inverse ALBDF $\tilde{C}_{j}^{l o c}=C\left(\tilde{F}_{j}^{r e f}, \phi_{l o c}, T_{r e f}\right)$

4) Calculate the local gray gas absorption coefficients $\kappa_{j}^{l o c}=N^{l o c} Y^{l o c} \sqrt{\tilde{C}_{j-1}^{l o c} \tilde{C}_{j}^{l o c}}, \kappa_{0}^{l o c}=0$

5) Local weights are calculated with the ALBDF at the local cross-sections $\tilde{C}_{j}^{\text {loc }}$ $a_{j}^{l o c}=F\left(\tilde{C}_{j}^{l o c}, \phi_{l o c}, T_{l o c}\right)-F\left(\tilde{C}_{j-1}^{l o c}, \phi_{l o c}, T_{l o c}\right), \quad a_{0}^{l o c}=F\left(\tilde{C}_{0}^{l o c}, \phi_{r e f}, T_{l o c}\right)$

Method I.2.1 In this new version of the reference approach the assumption of rank correlation allows calculation of the local absorption cross-sections without the help of a reference state. The method requires the generation of three ALBDFs.

1) Define the partition of the $F$-variable into reference values $\tilde{F}_{j}^{\text {ref }}, j=0,1, \ldots, n$ and $F_{j}^{\text {ref }}$, $j=1,2, \ldots, n$.

2) Calculate the local and the reference partition of the $C$-variable with the help of the inverse ALBDFs

$$
\begin{aligned}
& C_{j}^{\text {loc }}=C\left(F_{j}^{r e f}, \phi_{l o c}, T_{r e f}\right) \\
& \tilde{C}_{j}^{r e f}=C\left(\tilde{F}_{j}^{r e f}, \phi_{r e f}, T_{r e f}\right)
\end{aligned}
$$


3) Calculate the local gray gas coefficients $\kappa_{j}^{l o c}=N^{l o c} Y^{l o c} C_{j}^{l o c}, \quad \kappa_{0}^{l o c}=0$

4) Local weights are calculated using the ALBDF at the reference cross-sections $\tilde{C}_{j}^{\text {ref }}$ $a_{j}^{l o c}=F\left(\tilde{C}_{j}^{r e f}, \phi_{r e f}, T_{l o c}\right)-F\left(\tilde{C}_{j-1}^{r e f}, \phi_{r e f}, T_{l o c}\right), \quad a_{0}^{l o c}=F\left(\tilde{C}_{0}^{r e f}, \phi_{r e f}, T_{l o c}\right)$

Method I.2.2 This is a new method termed the Rank Correlated SLW model, distinguished by the fact that there is no need to specify a reference state or a reference absorption spectrum. It requires only two ALBDF distribution functions. It will be shown that this method is superior to all other versions of the SLW reference approach.

1) Define the partition of the $F$-variable into reference values $\tilde{F}_{j}^{\text {ref }}, j=0,1,2, \ldots, n$ and $F_{j}^{r e f}, j=1,2, \ldots, n$.

2) Find the local partition of the $C$-variable using the inverse ALBDF

$C_{j}^{l o c}=C\left(F_{j}^{r e f}, \phi_{l o c}, T_{r e f}\right)$

$\tilde{C}_{j}^{l o c}=C\left(\tilde{F}_{j}^{r e f}, \phi_{l o c}, T_{r e f}\right)$

3) Calculate the local gray gas coefficients $\kappa_{j}^{l o c}=N^{l o c} Y^{l o c} C_{j}^{l o c}, \quad \kappa_{0}^{l o c}=0$

4) Local weights are calculated with the help of the ALBDF at the local cross-sections $\tilde{C}_{j}^{\text {loc }}$ $a_{j}^{l o c}=F\left(\tilde{C}_{j}^{l o c}, \phi_{l o c}, T_{l o c}\right)-F\left(\tilde{C}_{j-1}^{l o c}, \phi_{l o c}, T_{l o c}\right), a_{0}^{l o c}=F\left(\tilde{C}_{0}^{l o c}, \phi_{l o c}, T_{l o c}\right)$

Because of the advantages this method presents a more detailed theoretical development is given in Section 3.7.

\subsection{Spectrum relationship at the local blackbody source temperature $T_{b}=T_{l o c}$}

Method II.1.1 Only two ALBDFs are generated for this new approach.

1) Start with subdivision of the $C$-variable into supplemental cross-sections $\tilde{C}_{j}^{r e f}, j=0,1, \ldots, n$.

2) Calculate the corresponding subdivision into local values $\tilde{F}_{j}^{\text {loc }}$ $\tilde{F}_{j}^{l o c}=F\left(\tilde{C}_{j}^{r e f}, \phi_{r e f}, T_{l o c}\right)$

3) Calculate the local supplemental cross-sections $\tilde{C}_{j}^{\text {loc }}$ $\tilde{C}_{j}^{l o c}=C\left(F_{j}^{l o c}, \phi_{l o c}, T_{l o c}\right)$

4) Calculate the local weights of gray gases $a_{j}^{l o c}=F\left(\tilde{C}_{j}^{r e f}, \phi_{r e f}, T_{l o c}\right)-F\left(\tilde{C}_{j-1}^{r e f}, \phi_{r e f}, T_{l o c}\right), \quad a_{0}^{l o c}=\tilde{F}_{0}^{l o c}$

Method II.1.2 This new method differs from Method II.1.1 only by the manner in which the local gray gas weights are calculated.

1) Start with subdivision of the $C$-variable into supplemental cross-sections $\tilde{C}_{j}^{r e f}, j=0,1, \ldots, n$.

2) Calculate the corresponding subdivision into local values $\tilde{F}_{j}^{l o c}$ $\tilde{F}_{j}^{l o c}=F\left(\tilde{C}_{j}^{r e f}, \phi_{r e f}, T_{l o c}\right)$

3) Calculate the local supplemental cross-sections $\tilde{C}_{j}^{\text {loc }}$ $\tilde{C}_{j}^{l o c}=C\left(F_{j}^{l o c}, \phi_{l o c}, T_{l o c}\right)$

4) The weights are calculated with the help of the local supplemental cross-sections $\tilde{C}_{j}^{\text {loc }}$ $a_{j}^{l o c}=F\left(\tilde{C}_{j}^{l o c}, \phi_{l o c}, T_{l o c}\right)-F\left(\tilde{C}_{j-1}^{l o c}, \phi_{l o c}, T_{l o c}\right)$ 
Substitution into this equation of $\tilde{C}_{j}^{l o c}=C\left(F_{j}^{l o c}, \phi_{l o c}, T_{l o c}\right)$ then yields

$$
\begin{aligned}
a_{j}^{l o c} & =F\left(C\left(\tilde{F}_{j}^{l o c}, \phi_{l o c}, T_{l o c}\right), \phi_{l o c}, T_{l o c}\right)-F\left(C\left(\tilde{F}_{j-1}^{l o c}, \phi_{l o c}, T_{l o c}\right), \phi_{l o c}, T_{l o c}\right) \\
& =\tilde{F}_{j}^{l o c}-\tilde{F}_{j-1}^{l o c} \\
& =F\left(\tilde{C}_{j}^{r e f}, \phi_{r e f}, T_{l o c}\right)-F\left(\tilde{C}_{j-1}^{r e f}, \phi_{r e f}, T_{l o c}\right)
\end{aligned}
$$

The calculation of the weights in this method is identical to that of Method II.1.1. Therefore, this approach produces results equivalent to Method II.1.1 (and the two equivalent methods may be hereafter referred to as II.1.1-2).

Method II.2.1 This reference approach method is also new. As seen from Figs. 8 and 9, this method requires three steps for calculation of the local absorption cross-sections, instead of multiple steps for the previous methods.

1) Define the partition of the $F$-variable into reference values $\tilde{F}_{j}^{r e f}, j=0,1,2, \ldots, n$ and $F_{j}^{r e f}, j=1,2, \ldots, n$.

2) Calculate the local and reference value supplemental absorption cross-sections $\tilde{C}_{j}^{\text {loc }}, \tilde{C}_{j}^{\text {ref }}$ and absorption cross-sections $C_{j}^{l o c}, C_{j}^{r e f}$ using the inverse ALBDF and the direct ALBDF

$\tilde{C}_{j}^{r e f}=C\left(\tilde{F}_{j}^{r e f}, \phi_{r e f}, T_{r e f}\right), \quad \tilde{F}_{j}^{l o c}=F\left(\tilde{C}_{j}^{r e f}, \phi_{r e f}, T_{l o c}\right)$

$C_{j}^{r e f}=C\left(F_{j}^{r e f}, \phi_{\text {ref }}, T_{\text {ref }}\right), \quad F_{j}^{\text {loc }}=F\left(C_{j}^{\text {ref }}, \phi_{\text {ref }}, T_{l o c}\right)$

$\tilde{C}_{j}^{l o c}=C\left(\tilde{F}_{j}^{l o c}, \phi_{l o c}, T_{r e f}\right), \quad C_{j}^{l o c}=C\left(F_{j}^{l o c}, \phi_{l o c}, T_{r e f}\right)$

3) Calculate the local gray gas coefficients
$\kappa_{j}^{l o c}=N^{l o c} Y^{l o c} C_{j}^{l o c}$,
$\kappa_{0}^{l o c}=0$

4) Local weights are calculated with the help of the ALBDF at the reference cross-sections $\tilde{C}_{j}^{\text {ref }}$ $a_{j}^{l o c}=F\left(\tilde{C}_{j}^{r e f}, \phi_{r e f}, T_{l o c}\right)-F\left(\tilde{C}_{j-1}^{r e f}, \phi_{r e f}, T_{l o c}\right), \quad a_{0}^{\text {loc }}=F\left(\tilde{C}_{0}^{r e f}, \phi_{r e f}, T_{l o c}\right)$

Method II.2.2 This method is technically similar to the improved FSK implementation reported in [11]. It differs formally from Method II.2.1 only by the manner in which the weights are calculated. However, in fact, it yields the same local weights as Method II.2.1.

1) Define the partition of the $F$-variable into reference values $\tilde{F}_{j}^{\text {ref }}, j=0,1, \ldots, n$ and $F_{j}^{\text {ref }}$, $j=1,2, \ldots, n$.

2) Calculate the local supplemental cross-sections $\tilde{C}_{j}^{l o c}$ and absorption cross-sections $C_{j}^{\text {loc }}$ using the inverse ALBDF and the direct ALBDF

$$
\begin{array}{ll}
\tilde{C}_{j}^{r e f}=C\left(\tilde{F}_{j}^{r e f}, \phi_{r e f}, T_{r e f}\right), & \tilde{F}_{j}^{l o c}=F\left(\tilde{C}_{j}^{r e f}, \phi_{r e f}, T_{l o c}\right) \\
C_{j}^{r e f}=C\left(F_{j}^{r e f}, \phi_{r e f}, T_{r e f}\right), & F_{j}^{l o c}=F\left(C_{j}^{r e f}, \phi_{r e f}, T_{l o c}\right) \\
\tilde{C}_{j}^{l o c}=C\left(\tilde{F}_{j}^{l o c}, \phi_{l o c}, T_{l o c}\right), & C_{j}^{l o c}=C\left(F_{j}^{l o c}, \phi_{l o c}, T_{l o c}\right)
\end{array}
$$

3) Calculate the local gray gas absorption coefficients

$$
\kappa_{j}^{l o c}=N^{l o c} Y^{l o c} C_{j}^{l o c}, \quad \kappa_{0}^{l o c}=0
$$

4) Local weights are calculated with the help of the ALBDF at the local cross-sections $\tilde{C}_{j}^{\text {loc }}$

$a_{j}^{l o c}=F\left(\tilde{C}_{j}^{l o c}, \phi_{l o c}, T_{l o c}\right)-F\left(\tilde{C}_{j-1}^{l o c}, \phi_{l o c}, T_{l o c}\right), \quad a_{0}^{l o c}=F\left(\tilde{C}_{0}^{l o c}, \phi_{r e f}, T_{l o c}\right)$

In fact, these weights are already predetermined by the values calculated at the reference crosssections $\tilde{C}_{j}^{\text {ref }}$, which makes this approach equivalent to Method II.2.1. Substitution of $C_{j}^{l o c}=C\left(F_{j}^{l o c}, \phi_{l o c}, T_{r e f}\right)$ into this equation calculated at step 2$)$, yields 


$$
\begin{aligned}
a_{j}^{l o c} & =F\left[C\left(F_{j}^{l o c}, \phi_{l o c}, T_{l o c}\right), \phi_{l o c}, T_{l o c}\right]-F\left[C\left(F_{j-l}^{l o c}, \phi_{l o c}, T_{l o c}\right), \phi_{l o c}, T_{l o c}\right] \\
& =\tilde{F}_{j}^{l o c}-\tilde{F}_{j-1}^{l o c} \\
& =F\left(C_{j}^{r e f}, \phi_{r e f}, T_{l o c}\right)-F\left(C_{j}^{r e f}, \phi_{r e f}, T_{l o c}\right)
\end{aligned}
$$

which is equivalent to step 4) of Method II.2.1. Therefore, this approach is equivalent to Method II.2.1 (and the two equivalent methods may be hereafter referred to as II.2.1-2).

\subsection{Spectral integration of the source term of the RTE}

It is instructive to investigate the spectral integration of the emission source term in the RTE for the reference approach methods presented here. Spectral integration of the emission source term for the medium at location $s$ with thermodynamic state $\phi_{g}=\phi(s)=\phi_{l o c}$ may be carried as follows:

$$
\int_{0}^{\infty} \kappa_{\eta}\left(\phi_{g}=\phi_{l o c}\right) I_{b \eta}\left(T_{l o c}\right) d \eta=\frac{\int_{0}^{\infty} \kappa_{\eta}\left(\phi_{g}=\phi_{l o c}\right) I_{b \eta}\left(T_{b}=T_{l o c}\right) d \eta}{I_{b}\left(T_{l o c}\right)} I_{b}\left(T_{l o c}\right)=\kappa_{P}\left(\phi_{l o c}, T_{l o c}\right) I_{b}\left(T_{l o c}\right)
$$

where $\kappa_{P}\left(\phi_{g}=\phi_{l o c}, T_{b}=T_{l o c}\right)$ is a modified Planck mean absorption coefficient [9] evaluated at the local state $\phi_{g}=\phi_{l o c}$ and the blackbody source temperature is the local temperature $T_{b}=T_{l o c}$. Therefore, for correct calculation of the source term in the RTE the local gas temperature $T_{g}$ and the emission source temperature $T_{b}$ must be the same local temperature of the medium $T_{g}=T_{b}=T_{l o c}$. Modelling of the spectral integration of the emission term by the SLW model over the gray gas intervals yields

$$
\int_{0}^{\infty} \kappa_{\eta}\left(\phi_{g}=\phi_{l o c}\right) I_{b \eta}\left(T_{b}\right) d \eta=I_{b}\left(T_{l o c}\right) \sum_{j=1}^{N} \kappa_{j}^{l o c}\left[F\left(\tilde{C}_{j}^{l o c}, \phi_{l o c}, T_{b}=T_{l o c}\right)-F\left(\tilde{C}_{j-1}^{l o c}, \phi_{l o c}, T_{b}=T_{l o c}\right)\right]
$$

The emission source term in the RTE is thus the product of the total blackbody intensity at the local temperature and a modified Planck mean absorption coefficient calculated with the same local gas and blackbody source temperature. Therefore, any valid model should calculate the gray gas weights with the ALBDF at the local gas thermodynamic state $\phi_{l o c}$ and the local source temperature $T_{b}=T_{\text {loc }}$ (i.e., the method should preserve the local Planck mean absorption coefficient). SLW modelling of the Planck mean absorption coefficient for the different reference approaches described above yields the following expressions:

For methods I.1.1, I.2.1, II.1.1-2, and II.2.1-2

$$
\sum_{j=1}^{N} \kappa_{j}^{l o c}\left[F\left(\tilde{C}_{j}^{r e f}, \phi_{r e f}, T_{b}=T_{l o c}\right)-F\left(\tilde{C}_{j-1}^{r e f}, \phi_{r e f}, T_{b}=T_{l o c}\right)\right]=\kappa_{P}\left(\phi_{r e f}, T_{l o c}\right)
$$

For methods I.1.2 and I.2.2

$$
\sum_{j=1}^{N} \kappa_{j}^{l o c}\left[F\left(\tilde{C}_{j}^{l o c}, \phi_{l o c}, T_{b}=T_{l o c}\right)-F\left(\tilde{C}_{j-1}^{l o c}, \phi_{l o c}, T_{b}=T_{l o c}\right)\right]=\kappa_{P}\left(\phi_{l o c}, T_{l o c}\right)
$$

Therefore, it may be seen that only Methods I.1.2 and I.2.2 correctly calculate the emission source term as $I_{b}\left(T_{l o c}\right) \kappa_{P}\left(\phi_{l o c}, T_{l o c}\right)$, thus preserving the local Planck mean absorption coefficient. No other approach preserves the spectrally integrated emission term at the local state, and without that conservation there is the potential for significant inaccuracy in modelling the source term in the 
spectrally integrated RTE where deviation of the local gas temperature from the reference temperature exists.

The differences in computational efficiency among the different methods should also be noted here. The number of steps needed to calculate the local gray gas absorption coefficients by the different methods is summarized as follows:

Methods I.2.1 and I.2.2: one step

Methods I.1.1, I.1.2, II.1.1, and II.1.2: two steps

Methods II.2.1 and II.2.2: three steps

\subsection{Outline of the Rank Correlated SLW model in non-uniform medium (Method I.2.2)}

Because the Rank Correlated SLW model (Method I.2.2) has significant advantages over other reference approaches the detailed development of the method is summarized in greater detail here. Consider the graphical relationships illustrated in Fig. 10. The methodology for determining the gray gas absorption coefficients and corresponding gray gas weights for the RTE are as follows:

1) Define the partition of the $F$-variable into reference values such that $F \in\left[F_{\min }, F_{\max }\right]$ :

$$
\tilde{F}_{0}^{r e f}=F_{\text {min }}, \tilde{F}_{j}^{r e f}=F_{\text {min }}+\left(F_{\text {max }}-F_{\text {min }}\right) \sum_{k=1}^{j} w_{k}, F_{j}^{r e f}=F_{\text {min }}+x_{j}\left(F_{\text {max }}-F_{\text {min }}\right), j=1,2, \ldots, n
$$

where $x_{j}>0$ are the positive abscissa and $w_{j}, j=1,2, \ldots, N$, are the corresponding weights of Gaussian-Legendre quadratures for integration over the interval $[-1,1]$.

2) Determine the local partition of the $C$-variable using the inverse ALBDF:

$C_{j}^{l o c}=C\left(F_{j}^{r e f}, \phi_{l o c}, T_{r e f}\right)$

$\tilde{C}_{j}^{l o c}=C\left(\tilde{F}_{j}^{r e f}, \phi_{l o c}, T_{r e f}\right)$

3) Calculate the local gray gas absorption coefficients:

$\kappa_{j}^{l o c}=N^{l o c} Y^{l o c} C_{j}^{l o c}, \quad \kappa_{0}^{l o c}=0$

4) Calculate the local gray gas weights with the help of the ALBDF at the local cross-sections $\tilde{C}_{j}^{l o c}$ : $a_{j}^{l o c}=F\left(\tilde{C}_{j}^{l o c}, \phi_{l o c}, T_{l o c}\right)-F\left(\tilde{C}_{j-1}^{l o c}, \phi_{l o c}, T_{l o c}\right), a_{0}^{l o c}=F\left(\tilde{C}_{0}^{l o c}, \phi_{l o c}, T_{l o c}\right)$

5) The parameters thus found define the local gray gas RTE in non-uniform medium:

$\frac{\partial I_{j}(s)}{\partial s}=-\kappa_{j}(s) I_{j}(s)+\kappa_{j}(s) a_{j}(s) I_{b}[T(s)]$

It should be noted that the current outline is presented for a single gas. The case of a mixture of gases with non-scattering soot particles can be treated as a single gas using the approach outlined in [6].

\subsection{Continuous limit of the Rank Correlated SLW model (Method I.2.2)}

With an increase in the number of gray gases the Generalized SLW model has been shown to approach the continuous exact limit [6]. To develop the limiting case, continuous exact limit of the Rank Correlated SLW model, Method I.2.2, one chooses $\tilde{F}_{j}^{\text {ref }}$ as the discrete independent variable, following which the gray gas intensity is defined as

$$
I_{j}(s, \mathbf{\Omega})=I\left(\tilde{F}_{j}^{r e f}, s, \mathbf{\Omega}\right) \Delta F_{j}^{r e f}
$$


where $\Delta F_{j}^{r e f}=\tilde{F}_{j}^{r e f}-\tilde{F}_{j-l}^{r e f}$ (see Fig. 11 for supporting graphical interpretation). Then the gray gas RTEs can be written in finite increment form

$$
\begin{aligned}
& \frac{\partial}{\partial s} I\left(\tilde{F}_{j}^{r e f}, s, \boldsymbol{\Omega}\right)=-\kappa_{j}^{l o c} I\left(\tilde{F}_{j}^{r e f}, s, \mathbf{\Omega}\right)+\kappa_{j}^{l o c} I_{b}\left(T_{l o c}\right) \frac{\Delta F_{j}^{l o c}}{\Delta F_{j}^{r e f}} \\
& =-\kappa_{j}^{l o c} I\left(\tilde{F}_{j}^{r e f}, s, \mathbf{\Omega}\right)+\kappa_{j}^{l o c} I_{b}\left(T_{l o c}\right) \frac{F\left(\tilde{C}_{j}^{l o c}, \phi_{l o c}, T_{l o c}\right)-F\left(\tilde{C}_{j-1}^{l o c}, \phi_{l o c}, T_{l o c}\right)}{\tilde{F}_{j}^{r e f}-\tilde{F}_{j-1}^{r e f}} \\
& =-\kappa_{j}^{l o c} I\left(\tilde{F}_{j}^{r e f}, s, \mathbf{\Omega}\right)+\kappa_{j}^{l o c} I_{b}\left(T_{l o c}\right) \frac{F\left[C\left(\tilde{F}_{j}^{r e f}, \phi_{l o c}, T_{r e f}\right), \phi_{l o c}, T_{l o c}\right]-F\left[C\left(\tilde{F}_{j-1}^{r e f}, \phi_{l o c}, T_{r e f}\right), \phi_{l o c}, T_{l o c}\right]}{\Delta F_{j}^{r e f}}
\end{aligned}
$$

Here, the gray gas absorption coefficients and their weights are calculated as outlined in the foregoing sections for Method I.2.2. In the limit, with an increase of number of gray gases, the increment $\Delta F_{j}^{\text {ref }} \rightarrow 0$; the discrete supplemental values approach the continuous variables $\tilde{F}_{j-1}^{r e f} \rightarrow F_{j} \rightarrow \tilde{F}_{j}^{r e f} \rightarrow \xi, \kappa_{j}^{l o c} \rightarrow \kappa(\xi)$; and the ratio of finite increments becomes a derivative of the ALBDF with respect to a continuous variable $\xi$ :

$$
\frac{\partial}{\partial s} I(\xi)=-\kappa(\xi, s) I(\xi)+\kappa(\xi, s) I_{b}[T(s)] \frac{\partial}{\partial \xi} F\left[C\left(\xi, \phi_{l o c}, T_{b}\right), \phi_{l o c}, T_{b}=T_{l o c}\right]
$$

After solving for $I(\xi)$, the total intensity can then be calculated by integration with respect to $\xi$

$$
I=\int_{0}^{\infty} I_{\eta} d \eta=\sum_{j=0}^{n} I\left(\tilde{F}_{j}^{r e f}, s, \mathbf{\Omega}\right) \Delta F_{j}^{r e f} \rightarrow I=\int_{0}^{l} I(\xi) d \xi
$$

This equation is a continuous limit of the Generalized SLW model in non-uniform media for Method I.2.2. The significance of Eq. (30) is that the scaling factor (the derivative) in the emission term includes no reference state, i.e., the term depends only on the local state $\phi_{l o c}$ (and arbitrary blackbody source temperature $\left.T_{b}\right)$. It should also be noted that the function $C\left(\xi, \phi_{l o c}\right)$ can be defined over an arbitrary measure, entirely eliminating the need of the blackbody source temperature $T_{b}$.

\section{VALIDATION}

This section illustrates the use of the reference approaches presented above in example problems designed to test their accuracy over a range of problem parameters.

\subsection{Example 1 Hot layer - cold layer system}

The first example considered here is commonly used as a rigorous standard test case for comparison of the performance of different spectral models in nonisothermal gas media $[8,11]$. Consider a onedimensional system of two adjacent gas layers with different fixed values of gas temperature, $T_{\text {hot }}=2000 \mathrm{~K}$ and $T_{\text {cold }}=300 \mathrm{~K}$, as shown in Fig. 12a. The system is bounded by walls which are assumed to be black and cold. The gas in both layers is a homogenous mixture of water vapor with mole fraction $Y_{\mathrm{H}_{2} \mathrm{O}}=0.2$, and carbon dioxide with $Y_{\mathrm{CO}_{2}}=0.1$. The objective is to predict the directional total radiative flux at the exit of the cold layer $q(L)$ as a function of the thickness of the cold layer, $L_{\text {cold }}$. This example is known to be challenging for spectral modelling in gases because of the strong temperature gradient between the two layers. The SLW model analytical solution for the directional total radiative flux in the two-layer system bounded by black cold walls has been presented previously as [4] 
$q(L)=2 \sigma \sum_{j=0}^{n}\left[-a_{j}^{(I)} T_{1}^{4} E_{3}\left(\kappa_{j}^{(I)} L_{1}+\kappa_{j}^{(2)} L_{2}\right)+\left(a_{j}^{(l)} T_{1}^{4}-a_{j}^{(2)} T_{2}^{4}\right) E_{3}\left(\kappa_{j}^{(2)} L_{2}\right)+a_{j}^{(2)} T_{2}^{4} / 2\right]$

where $T_{1}=T_{\text {hot }}, \quad T_{2}=T_{\text {cold }}, \quad L_{1}=L_{\text {hot }}=0.5 \mathrm{~m}, L_{2}=L_{\text {cold }}, \quad L=L_{1}+L_{2}$, and the parenthetical superscripts refer to the corresponding layer. In this equation, $\kappa_{j}^{(k)}$ and $a_{j}^{(k)}$ are the local values of the gray gas absorption coefficient and their local weights, respectively, which must be found according the reference approach selected. Tabulated data for the ALBDF [5] were used in the calculations (rather than mathematical correlations). Line-by-line benchmark predictions were generated using the same database as that from which the ALBDF data were generated. Figure $12 \mathrm{~b}$ shows predictions of the normalized total flux at the exit of the cold layer $q(L) / E_{b}\left(T_{h o t}\right)=q(L) / \sigma T_{h o t}^{4}$ obtained using the different reference approaches presented previously. In Methods I.1.1 and I.2.1 the local weights are calculated with the reference supplemental cross-sections $\tilde{C}_{j}^{\text {ref }}$. Figure $12 \mathrm{~b}$ shows that predictions for these two methods are nearly identical, deviating significantly from the line-by-line solution, with increasing error as the thickness of the cold layer is increased. Recall that neither of these methods preserve the Planck mean absorption coefficient, $\kappa_{P}$, in the spectral integration of the RTE. Predictions using Methods I.1.2 and I.2.2 (both of which preserve $\kappa_{P}$ ), in which the local weights are calculated based on local supplemental cross-sections $\tilde{C}_{j}^{l o c}$, are considerably more accurate. As seen in Fig. $12 \mathrm{~b}$ for this example, all approaches, Methods II.1.1-2 and II.2.1-2, yield identical results.

\subsection{Example 2 Isothermal dual layer system}

This example is intended to confirm that if the gas spectra at different states are rigorously scaled (i.e., in the calculation of the absorption coefficient $\kappa_{\eta}=N Y C_{\eta}(T)$ only the mole fraction changes), then all versions of the reference approach presented here should produce accurate predictions in non-uniform medium.

Consider a system of two adjacent plane-parallel gas layers filled with $\mathrm{CO}_{2}$ at the same temperature $T=1000 \mathrm{~K}$ with different but fixed values of gas mole fraction, $Y_{1}=0.4$ (thick layer) and $Y_{1}=0.1$ (thin layer). The system is bounded by black and cold walls. Because the two layers differ only in mole fraction of the same gas, the gas absorption cross-sections according to the spectral model adopted are exactly the same for both layers (air-broadening and self-broadening are assumed to be the same for $\mathrm{CO}_{2}$ ), and the absorption coefficient will be scaled only by the mole fraction. Therefore, all versions of the reference approach should theoretically yield the same prediction of the exiting radiative flux, and should match the line-by-line result. The solution of this problem is obtained by the same analytical method used in Example 1.

Figure 13a shows the prediction of the normalized total flux at the exit of the thin layer $q(L) / E_{b}(T)=q(L) / \sigma T^{4}$ as a function of the thickness of the thinner layer $L_{2}$ obtained using the different reference approaches. Figure $13 \mathrm{~b}$ shows the normalized relative error. For this example featuring perfectly scaled spectra, all approaches yield nearly identical, accurate results. The relative error is generally on the order of $0.3 \%$ or less except at low $L_{2}$ for methods II.1.1-2, II.2.-1, and I.1.2. Although the relative error in prediction of the exiting flux for all methods is very low as expected for this example, the Rank Correlated SLW reference approach, Method I.2.2, (which does not require the specification of a reference state) exhibits noticeably better accuracy. The likely reason for the lower error of Method I.2.2 is that this method requires the evaluation of only two ALBDF distribution functions, unlike the other approaches which require the evaluation of three 
distribution functions. This example demonstrates that indeed, if spectra are perfectly scaled then all proposed reference approaches yield accurate prediction of radiative transfer in non-uniform medium.

\subsection{Example 3 Non-isothermal, non-homogeneous layer of $\mathrm{H}_{2} \mathrm{O}$}

Consider now the prediction of the divergence of total net radiative flux in a plane-parallel layer of thickness $L=1.0 \mathrm{~m}$ filled with a gas at total pressure $p=1.0 \mathrm{~atm}$ with a parabolic temperature profile, $T(x)=4000 x(L-x) / L^{2}+800, K$, and imposed wall temperatures $T(0)=800 \mathrm{~K}$ and $T(L)=800 \mathrm{~K}$. The layer is filled with water vapor of spatially variable mole fraction given by $Y_{\mathrm{H}_{2} \mathrm{O}}(x)=0.8 x(L-x) / L^{2}+0.12$. Predictions of the total divergence of the net radiative flux using the Generalized SLW reference approaches Methods I.1.1 and I.2.2 and the LBL solution, obtained with the same spectral database [5], are shown in Fig. 14. Method I.1.1 is the original SLW reference approach, and Method I.2.2 is the simpler, more efficient Rank Correlated SLW model (Method I.2.2) which does not require specification of a reference state and preserves the emission term in the RTE. For this example both the original (I.1.1) and the improved reference approach (I.2.2) yield good accuracy relative to the line-by-line benchmark prediction, which is not unexpected in a problem with smooth, continuous variation of temperature and concentration with moderate local gradients.

\subsection{Example 4 Triangular temperature profile $\mathrm{H}_{2} \mathrm{O}$}

The Rank Correlated SLW model requires the specification of only the blackbody source temperature $T_{b}$ (which has been previously stated is only a matter of mathematical convenience), whereas the other SLW reference approaches need a reference state which includes both $T_{g}=T_{r e f}$ and $T_{b}=T_{r e f}$. Their roles are different: the reference state defines the reference absorption coefficient which must then be scaled to any other local state; the source temperature defines the Planck blackbody distribution which is used simply for reordering of the absorption cross-section to any local state. The same measure (and therefore, the same source temperature $T_{b}$ ) should be used for reordering at all local states in the medium.

As has been stated previously, theoretically, the choice of the blackbody source temperature $T_{b}$ should be unimportant. To demonstrate the sensitivity of the choice of $T_{r e f}$ and $T_{b}$ the following example known to be a challenging problem from the research reported in ref. [8] is considered. Consider a plane-parallel layer of thickness $L=0.3 \mathrm{~m}$ filled with a gas at total pressure $p=1.0 \mathrm{~atm}$ with the triangular temperature profile shown in Fig.15a, and imposed black walls at temperature $T(0)=T(L)=500 \mathrm{~K}$. The layer is filled with water vapor at uniform mole fraction $Y_{H_{2} O}(x)=0.1$. Line-by-line benchmark predictions are made, along with predictions using the original SLW reference approach (Method I.1.1) and the Rank Correlated SLW model (Method I.2.2). The same grid resolution (81 equally spaced points) was used in both the line-by-line and the SLW model predictions for both methods.

Predictions of the divergence of the total net radiative flux with the original SLW reference approach (Method I.1.1) and the Rank Correlated SLW model (Method I.2.2) are shown in Figs. $15 \mathrm{~b}$ and $15 \mathrm{c}$, respectively, along with the line-by-line predictions. The values for $T_{b}$ and $T_{\text {ref }}$ (where required) are specified to be identical for these simulations, and for comparison two different values are investigated: i) $T_{b}=T_{\text {ref }}=T_{\text {ave }}$ where $T_{\text {ave }}$ is the volumetrically averaged temperature of the 
layer, and $i$ ) $T_{b}=T_{\text {ref }}=T_{\max }$ where $T_{\max }$ is the maximum temperature of the layer. As can be seen in Fig. 15b, prediction of the total divergence of the net radiative flux is very sensitive to the reference temperature for the original SLW reference approach (Method I.1.1). By contrast, Fig. 15c shows that predictions for the Rank Correlated SLW model (Method I.2.2) are virtually insensitive to the choice of reference blackbody source temperature. Further, the Rank Correlated SLW model produces significantly more accurate predictions.

\subsection{Example 5 Non-isothermal, homogeneous layer of $\mathrm{H}_{2} \mathrm{O}$}

Subdivision into gray gases in the SLW model is usually performed using logarithmically spaced supplemental absorption cross-sections. This subdivision generally provides good accuracy with 8 12 gray gases. The new Rank Correlated SLW model can use fixed subdivision of the $F$-variable somewhat more efficiently for numerical integration. For example, positive Gauss-Legendre quadratures have been used with good results and significant computational economy. Therefore, subdivision into gray gases can be more efficient with the Rank Correlated SLW model.

The next example compares prediction by the SLW reference approach and the Rank Correlated SLW model of the radiation transfer in a homogeneous layer of water vapor with significant spatial temperature variation. The focus in this example is on the dependence of simulation results on the sensitivity of predictions in both methods to the number of gray gases used in the model. Consider prediction of the divergence of total net radiative flux in a plane-parallel layer of thickness $L=2.0 \mathrm{~m}$ filled with a water vapor at total pressure $p=1.0 \mathrm{~atm}$ with a sinusoidal temperature profile, $T(x)=1000.0+500 \cos (\pi x / L), K$, and black walls at temperature $T(0)=1500 \mathrm{~K}$ and $T(L)=500 \mathrm{~K}$. The layer is filled with water vapor of spatially uniform mole fraction $Y_{\mathrm{H}_{2} \mathrm{O}}(x)=0.1$. Predictions are shown in Fig. 16 for the original SLW reference approach (Method I.1.1) and the Rank Correlated SLW model (Method I.2.2), along with the line-by-line benchmark. The results show that the SLW reference approach (Method I.1.1) is quite sensitive to the number of gray gases used in the simulation, and significant error is observed as the number of gray gases is reduced. The method shows very good accuracy for $n>5$ in this problem. By contrast, the Rank Correlated SLW model (Method I.2.2) is insensitive to the number of gray gases used in the prediction, and the predictions show good accuracy with as few as 3 gray gases. The improved accuracy and insensitivity to the number of gray gases for the Rank Correlated SLW model is a critical finding, further underlining the advantages to this new method.

\section{CONCLUSIONS}

A range of possibilities for treatment of gas radiative transfer in non-uniform media is explored using the framework of the Generalized SLW model. It is shown that in total, eight different reference approaches are possible, whereas only three have been previously reported in the literature. The development reveals a version of the Generalized SLW reference approach (termed the Rank Correlated SLW model, Method I.2.2) that does not require specification of a gas reference thermodynamic state, and which preserves the spectrally integrated emission (Planck mean absorption coefficient) in the RTE. Construction of the Rank Correlated SLW model requires only two ALBDFs, and subdivision into gray gases can be performed using any accurate integration scheme, e.g., Legendre-Gaussian quadratures. Consequently, this new reference approach appears to have significant advantages over previously published approaches in non-homogeneous gas media. Predictions for sample problems reveal the method's accuracy and robustness. 


\section{Acknowledgement}

This work was partially supported by Institut National des Sciences Appliquées de Lyon, France.

\section{REFERENCES}

1. M.K. Denison, B.W. Webb, "A spectral line based weighted-sum-of-gray-gases model for arbitrary RTE solvers,” ASME J. Heat Transfer, vol.115, pp. 1004-1012, 1993.

2. M.K. Denison, B.W. Webb, "The spectral line based weighted-sum-of-gray-gases model in nonisothermal non-homogeneous media," ASME J. Heat Transfer, vol.117, pp. 359-365, 1995.

3. V.P. Solovjov, Spectral Line-Based Weighted-Sum-of-Gray-Gases Modeling of Radiative Transfer in Multicomponent Mixtures of Hot Gases, PhD Dissertation, BYU, Provo, Utah, 1998.

4. V.P. Solovjov, B.W. Webb, "Multilayer modeling of radiative transfer by SLW and CW methods in non-isothermal gaseous media," J. Quant. Spectr. Radiat. Transfer, vol.109, pp. 245257, 2008.

5. J.T. Pearson, B.W. Webb, V.P. Solovjov, J. Ma, "Efficient representation of the absorption line blackbody distribution function for $\mathrm{H}_{2} \mathrm{O}, \mathrm{CO}_{2}$, and $\mathrm{CO}$ at variable temperature, mole fraction, and total pressure," J. Quant. Spectr. Radiat. Transfer, vol. 138, pp. 82-96, 2014.

6. V.P. Solovjov, F. Andre, D. Lemonnier, B.W. Webb, "The Generalized SLW model," Eurotherm Conference 105: Computational Thermal Radiation in Participating Media V, Journal of Physics: Conference Series, 012022 vol. 676, pp.1-36, 2016.

7. F. Andre, R. Vaillon, "The spectral-line moment-based (SLMB) modeling of the wide band and global blackbody-weighted transmission function and cumulative distribution function of the absorption coefficient in uniform media," J. Quant. Spectr. Radiat. Transfer, vol. 109, pp. 24012416, 2008.

8. L. Pierrot, Ph. Rivière, A. Soufiani, J. Taine, "A fictitious-gas-based absorption distribution function global model for radiative transfer in hot gases," J. Quant. Spectr. Radiat. Transfer, vol. 62, pp. 609-624, 1999.

9. M.F. Modest, Radiative Heat Transfer, 3rd Ed., Academic Press, New York, 2013.

10. M.F. Modest, H. Zhang, "The full-spectrum correlated- $k$ distribution and its relationship to the weighted-sum-of-gray-gases method," Proc. of the 2000 IMECE HTD-366-1 Orlando FL, ASME New York 75-84, 2000.

11. J. Cai, M.F. Modest, "Improved full-spectrum $k$-distribution implementation for inhomogeneous media using a narrow-band database," J. Quant. Spectr. Radiat. Transfer, vol. 141, pp. 65-72, 2014.

12. H. Chu, F. Liu, J.-L. Consalvi. "Relationship between the spectral line based weighted-sum-ofgray-gases model and the full spectrum $k$-distribution model," J. Quant. Spectr. Radiat. Transfer, vol. 143, pp. 111-120, 2014.

13. R.M. Goody, Y.L. Yung. Atmospheric Radiation, Clarendon Press, Oxford, 1989. 


\section{Appendix 1. Preservation of spectral intervals of integration in the rank correlated method.}

This appendix demonstrates theoretically that the Rank Correlated SLW model preserves the spectral intervals of integration, which is critical to ensure that there are no Leibnitz terms in the spectrally integrated RTE, Eqs. (10) and (11). Let $C_{\eta}\left(\phi_{1}\right)$ and $C_{\eta}\left(\phi_{2}\right)$ be rank correlated absorption cross-sections at states $\phi_{1}$ and $\phi_{2}$. Correspondingly, by the definition of rank correlation, for any $\eta^{\prime} \in(0, \infty), \quad\left\{\eta: C_{\eta}\left(\phi_{1}\right)<C_{\eta^{\prime}}\left(\phi_{1}\right)\right\}=\left\{\eta: C_{\eta}\left(\phi_{2}\right)<C_{\eta^{\prime}}\left(\phi_{2}\right)\right\}$. Let $C\left(\xi, \phi_{1}, T_{b}\right), \xi \in(0,1)$ be the reordered absorption cross-section of $C_{\eta}\left(\phi_{1}\right)$, and let $C\left(\xi, \phi_{2}, T_{b}\right), \xi \in(0,1)$ be the reordered absorption cross-section of $C_{\eta}\left(\phi_{2}\right)$. Then for any fixed number $\xi \in(0,1)$,

$$
\left\{\eta: C_{\eta}\left(\phi_{1}\right)<C\left(\xi, \phi_{1}, T_{b}\right)\right\}=\left\{\eta: C_{\eta}\left(\phi_{2}\right)<C\left(\xi, \phi_{2}, T_{b}\right)\right\}
$$

Note that the reordered absorption cross-section $C=C\left(\xi, \phi, T_{b}\right)$ is the inverse of the ALBDF $\xi=F\left(C, \phi_{2}, T_{b}\right)$. The proof may be stated as follows. Fix an arbitrary number $\xi$ in the interval between 0 and $1, \xi \in(0,1)$. Calculate the value $C_{1}=C\left(\xi, \phi_{1}, T_{b}\right)$, which is the inverse ALBDF $\xi=F\left(C_{l}, \phi_{1}, T_{b}\right)$, and define wavenumber intervals $\Delta_{l}=\left\{\eta: C_{\eta}\left(\phi_{l}\right)<C_{l}\right\}$. Let $\eta^{\prime}$ be any wavenumber such that $C_{\eta^{\prime}}\left(\phi_{1}\right)=C_{1}$. At least one such value $\eta^{\prime}$ should exist, because from the definition of the distribution function,

$0 \leq C\left(\xi, \phi_{1}, T_{b}\right)<\max _{0<\eta<\infty} C_{\eta}\left(\phi_{1}\right)$

for any $\xi \in(0,1)$. Consequently, consistent with the assumption of rank correlated spectra, $C_{2}=C_{\eta^{\prime}}\left(\phi_{2}\right)$ is such that

$$
\Delta_{2}=\left\{\eta: C_{\eta}\left(\phi_{2}\right)<C_{2}\right\}=\Delta_{1}=\left\{\eta: C_{\eta}\left(\phi_{1}\right)<C_{1}\right\}
$$

The values of the ALBDF at $C=C_{1}, \phi=\phi_{1}$ and at $C=C_{2}, \phi=\phi_{2}$ with the same blackbody source temperature $T_{b}$ should be equal because they represent the same fraction of the Planck blackbody emissive power:

$$
F\left(C_{2}, \phi_{2}, T_{b}\right)=\frac{1}{E_{b}\left(T_{b}\right)} \int_{\Delta_{2}} E_{b \eta}\left(T_{b}\right) d \eta=\frac{1}{E_{b}\left(T_{b}\right)} \int_{\Delta_{l}} E_{b \eta}\left(T_{b}\right) d \eta=F\left(C_{1}, \phi_{1}, T_{b}\right)=\xi
$$

The value of $C_{2}$ satisfying this equation is unique, because $F\left(C, \phi_{2}, T_{b}\right)$ is a strictly increasing function of $C$. Therefore, $C_{2}=C\left(\xi, \phi_{2}, T_{b}\right)$, and the desired relationship Eq. (A1.2) holds:

$$
\left\{\eta: C_{\eta}\left(\phi_{1}\right)<C\left(\xi, \phi_{1}, T_{b}\right)=C_{1}\right\}=\left\{\eta: C_{\eta}\left(\phi_{2}\right)<C\left(\xi, \phi_{2}, T_{b}\right)=C_{2}\right\}
$$

The implication of this relation is that the Rank Correlated SLW model, Method I.2.2, preserves the gray gas spectral intervals for any gas thermodynamic state, and therefore, spectral integration of the RTE does not generate Leibnitz terms.

\section{Appendix 2. Preservation of spectral intervals of integration in methods with correlation at $T_{b}=T_{l o c}$}

This appendix shows theoretically that reference methods with correlation at $T_{b}=T_{l o c}$ preserve the spectral intervals of integration.

Consider Method II.1.1, which is a version with spectral correlation at the local blackbody source temperature $T_{b}=T_{l o c}$. Choose the reference state $\phi_{\text {ref. }}$ If one subdivides the $C$-variable into 
$\tilde{C}_{j}^{r e f}=C_{\min }\left(C_{\max } / C_{\min }\right)^{j / n}, j=0,1, \ldots, n$, the intersections of $\tilde{C}_{j}^{r e f}$ and $\tilde{C}_{j-1}^{r e f}$ with $C_{\eta}\left(\phi_{r e f}\right)$ define the wavenumber intervals:

$\Delta_{j}^{r e f}=\left\{\eta: \tilde{C}_{j-1}^{r e f}<C_{\eta}\left(\phi_{r e f}\right)<\tilde{C}_{j}^{r e f}\right\}$

These intervals are fixed because they are defined by fixed reference quantities. The assumption of a correlated spectrum yields that for the absorption cross-section $C_{\eta}\left(\phi_{l o c}\right)$ at any local state $\phi_{l o c}$ there exist local supplemental cross-sections $\tilde{C}_{j}^{l o c}$ and $\tilde{C}_{j-1}^{\text {loc }}$ such that

$F\left(\tilde{C}_{j}^{r e f}, \phi_{r e f}, T_{b}=T_{l o c}\right)=F\left(\tilde{C}_{j}^{l o c}, \phi_{l o c}, T_{b}=T_{l o c}\right)$

$F\left(\tilde{C}_{j-1}^{r e f}, \phi_{r e f}, T_{b}=T_{l o c}\right)=F\left(\tilde{C}_{j-1}^{l o c}, \phi_{l o c}, T_{b}=T_{l o c}\right)$

Therefore, the gray gas intervals at the local state are the same as the gray gas intervals at the reference state

$\Delta_{j}^{l o c}=\left\{\eta: \tilde{C}_{j-1}^{l o c}<C_{\eta}\left(\phi_{l o c}\right)<\tilde{C}_{j}^{l o c}\right\}=\Delta_{j}^{r e f}$

Consequently, this approach preserves the spectral intervals of integration for all locations.

\section{Appendix 3. Co-monotonicity of rank correlated absorption cross-sections.}

This appendix demonstrates rigorously that if spectra at two different thermodynamic states $C_{\eta}\left(\phi_{1}\right)$ and $C_{\eta}\left(\phi_{2}\right)$ are rank correlated, then for any two prescribed values $C_{1}$ and $C_{2}$, either the set of all wavenumbers $\eta$ such that $C_{\eta}\left(\phi_{1}\right)<C_{1}$ is a subset of the set of all wavenumbers $\eta$ for which $C_{\eta}\left(\phi_{2}\right)<C_{2}$, or vice versa. If the spectra $C_{\eta}\left(\phi_{1}\right)$ and $C_{\eta}\left(\phi_{2}\right)$ are rank correlated, then for any two prescribed values $C_{1}$ and $C_{2}$ such that $C_{\min , 1}<C_{1}<C_{\max , 1}$ and $C_{\operatorname{min,2}}<C_{2}<C_{\max , 2}$, either

$\left\{\eta: C_{\eta}\left(\phi_{1}\right)<C_{1}\right\} \subseteq\left\{\eta: C_{\eta}\left(\phi_{2}\right)<C_{2}\right\}$

or

$\left\{\eta: C_{\eta}\left(\phi_{1}\right)<C_{1}\right\} \supseteq\left\{\eta: C_{\eta}\left(\phi_{2}\right)<C_{2}\right\}$

First, the obvious fact may be formulated that for an absorption cross-section $C_{\eta}(\phi)$ at an arbitrary state $\phi$, if $S_{1}<S_{2}$, then $\left\{\eta: C_{\eta}(\phi)<S_{1}\right\} \subseteq\left\{\eta: C_{\eta}(\phi)<S_{2}\right\}$. Now let $\eta^{\prime}$ be any wavenumber such that $C_{\eta^{\prime}}\left(\phi_{1}\right)=C_{1}$. At least one such value $\eta^{\prime}$ should exist, because $C_{\min , l}<C_{1}<C_{\max , l}$. If one defines wavenumber intervals $\Delta_{l}=\left\{\eta: C_{\eta}\left(\phi_{1}\right)<C_{1}\right\}$, then according to the assumption of rank correlated spectra, $X=C_{\eta^{\prime}}\left(\phi_{1}\right)$ is such that

$\Delta_{2}=\left\{\eta: C_{\eta}\left(\phi_{2}\right)<X\right\}=\Delta_{1}=\left\{\eta: C_{\eta}\left(\phi_{1}\right)<C_{1}\right\}$

The prescribed value $C_{2}$ is, according to established fact, either $X<C_{2}$, and then

$\left\{\eta: C_{\eta}\left(\phi_{1}\right)<C_{1}\right\}=\left\{\eta: C_{\eta}\left(\phi_{2}\right)<X\right\} \subseteq\left\{\eta: C_{\eta}\left(\phi_{2}\right)<C_{2}\right\}$

or $X>C_{2}$, from which it follows that

$\left\{\eta: C_{\eta}\left(\phi_{1}\right)<C_{1}\right\}=\left\{\eta: C_{\eta}\left(\phi_{2}\right)<X\right\} \supseteq\left\{\eta: C_{\eta}\left(\phi_{2}\right)<C_{2}\right\}$ 

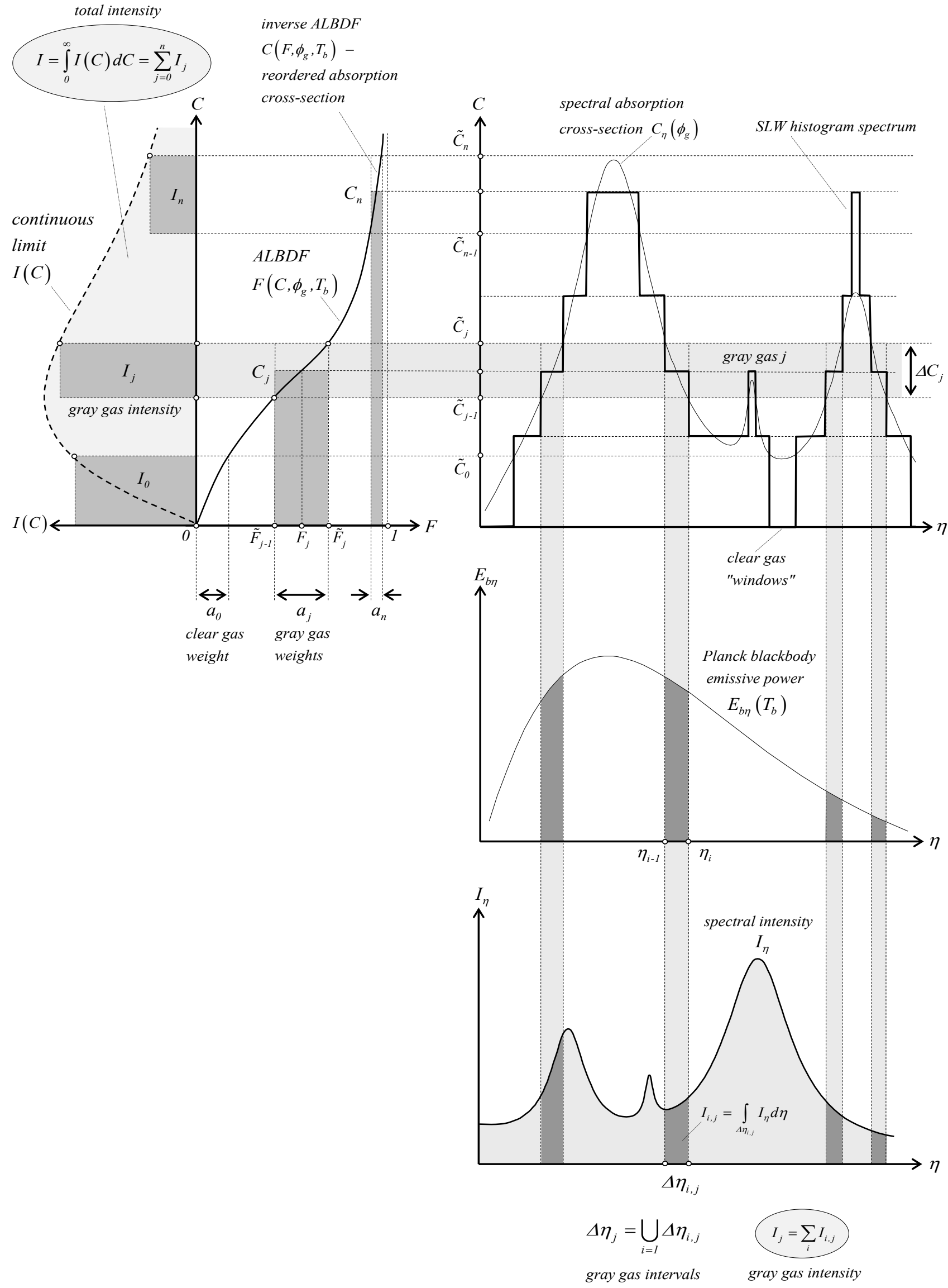

Figure 1. Geometric interpretation of the SLW model in uniform medium 

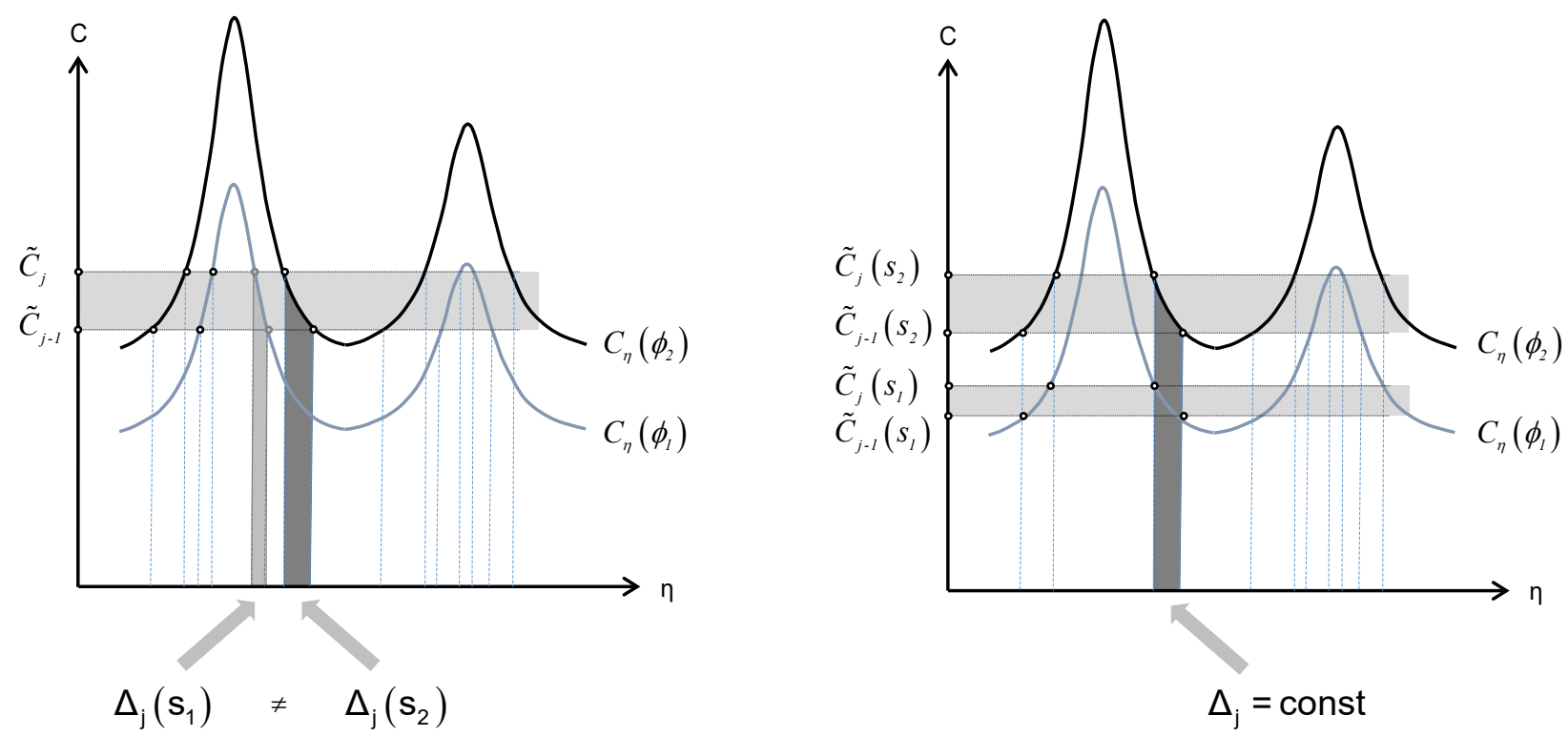

Figure 2. Gray gas wavenumber intervals $\Delta_{j}(s)$ in non-uniform media: a) fixed supplemental cross-sections $\tilde{C}_{j}=$ const - variable intervals $\Delta_{j}(s)$, and b) variable supplemental cross-sections $\tilde{C}_{j}(s)$ - fixed intervals $\Delta_{j}=$ constant . 


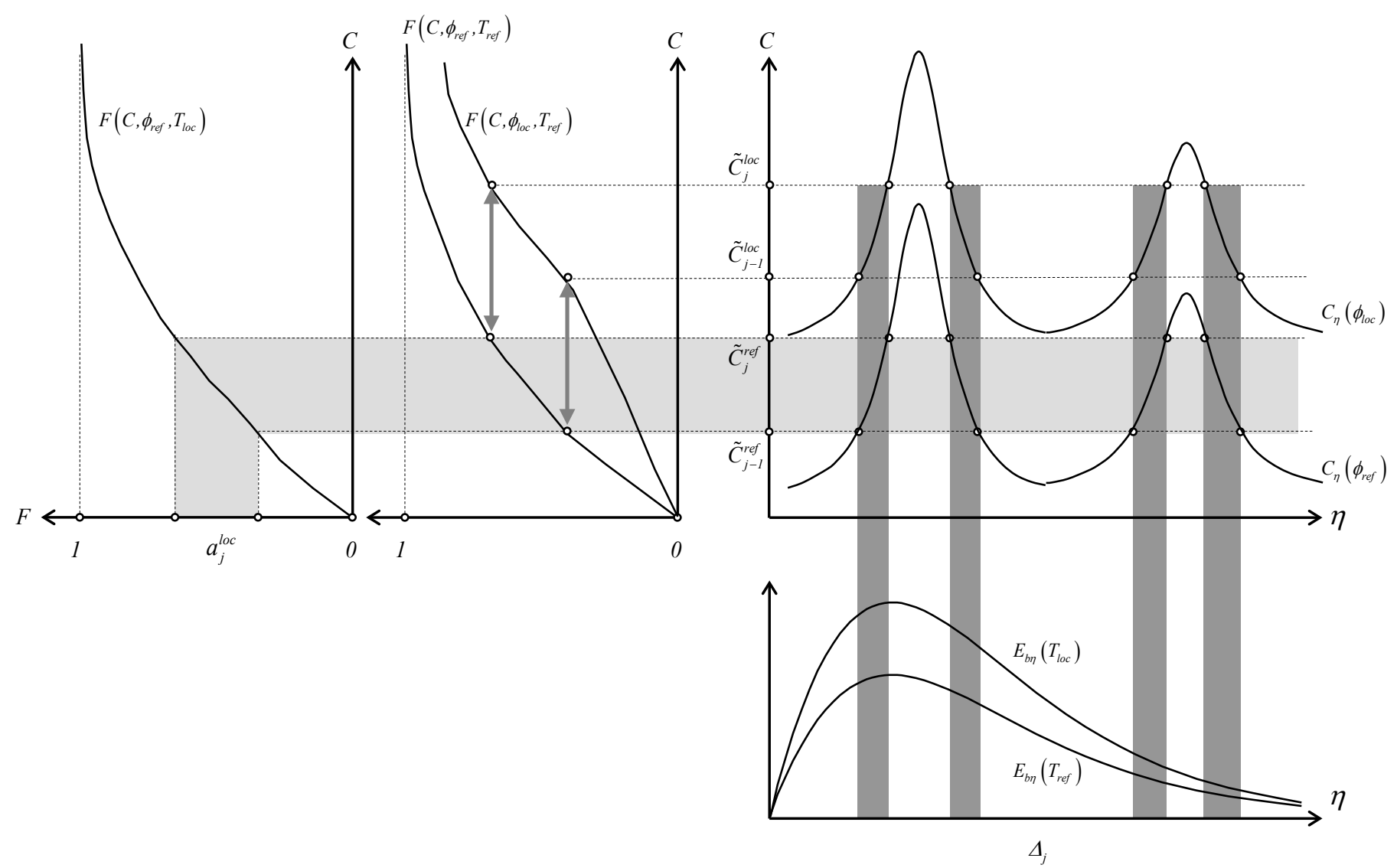

Figure 3. Geometrical interpretation of the SLW reference approach. 


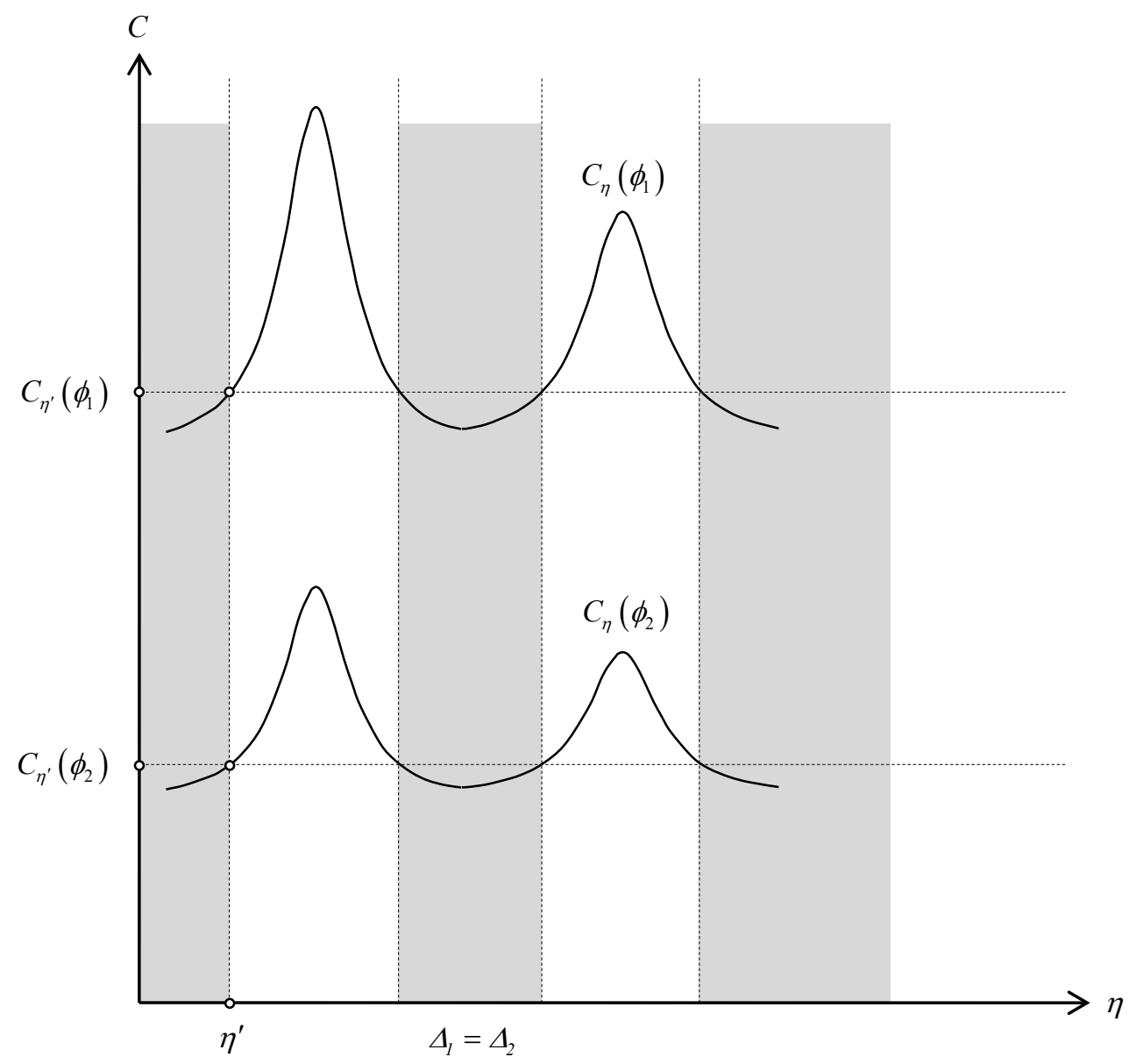

Figure 4. Rank correlated gas absorption cross-section $C_{\eta}(\phi)$ at two different thermodynamic states $\phi_{1}$ and $\phi_{2}$. 


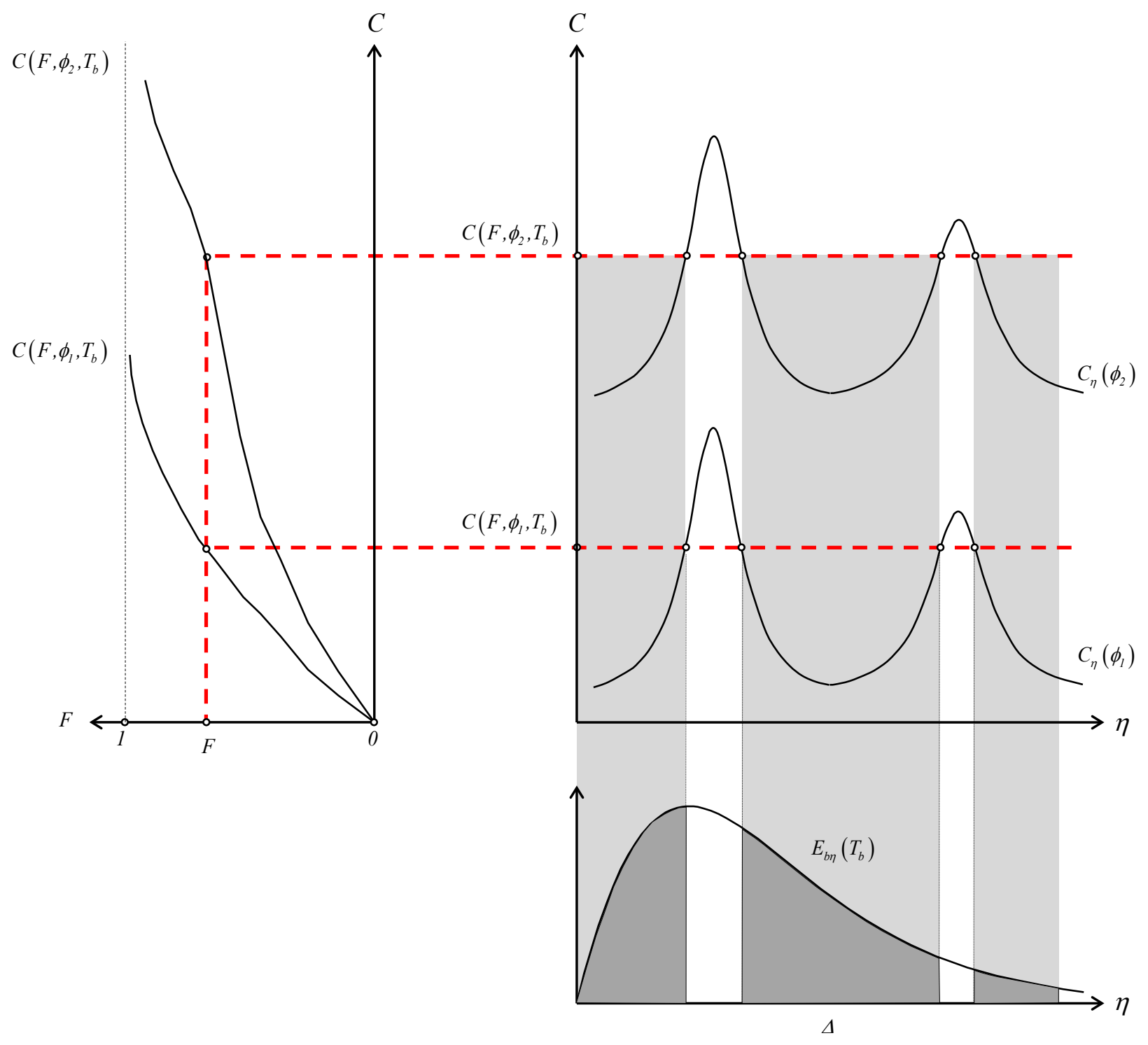

Figure 5. Rank correlated reordered absorption cross-section. 


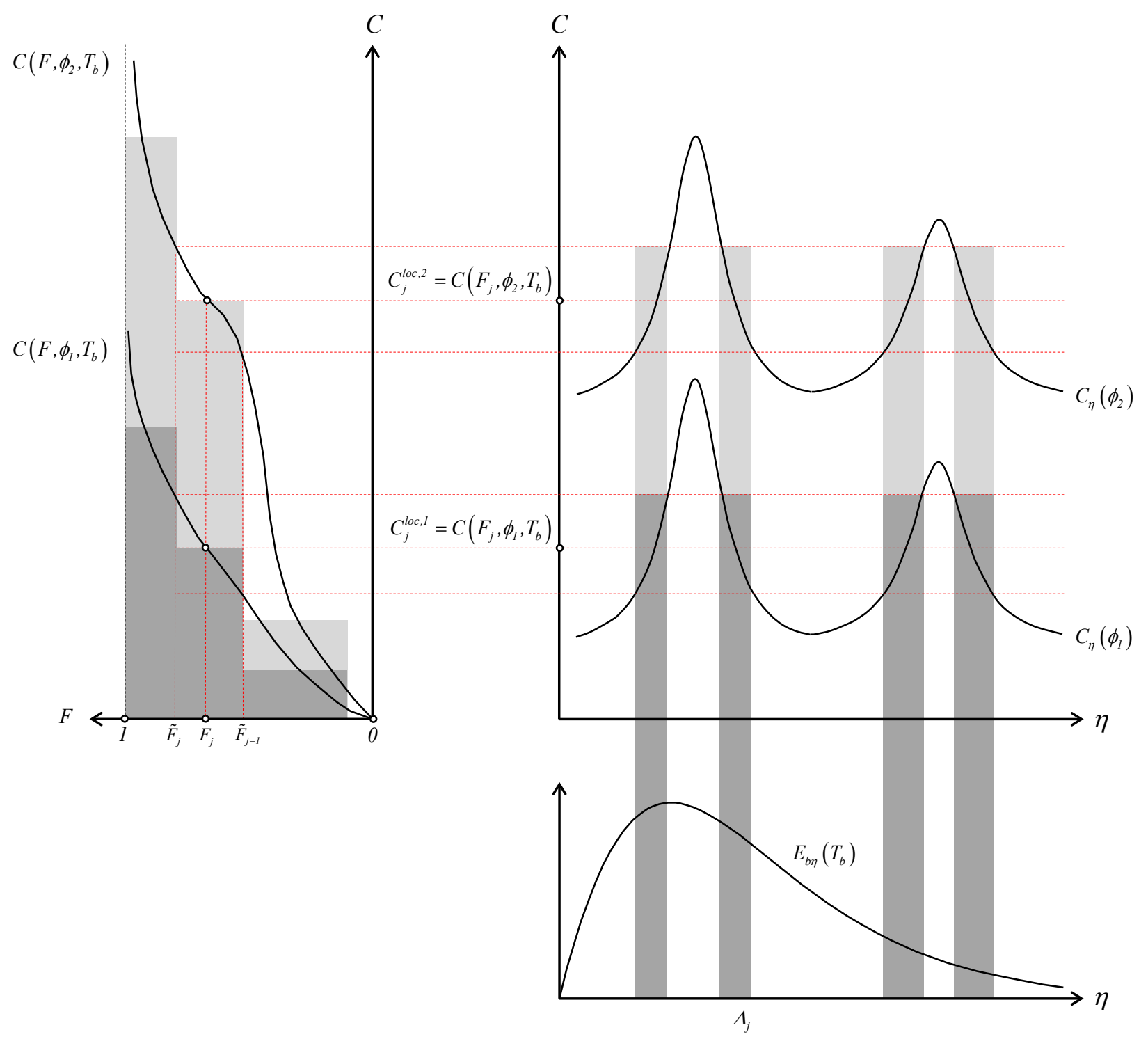

Figure 6. Rank correlated reordered absorption cross-sections $C\left(\tilde{F}_{j}, \phi_{l o c}, T_{b}\right)$ of the SLW model. 


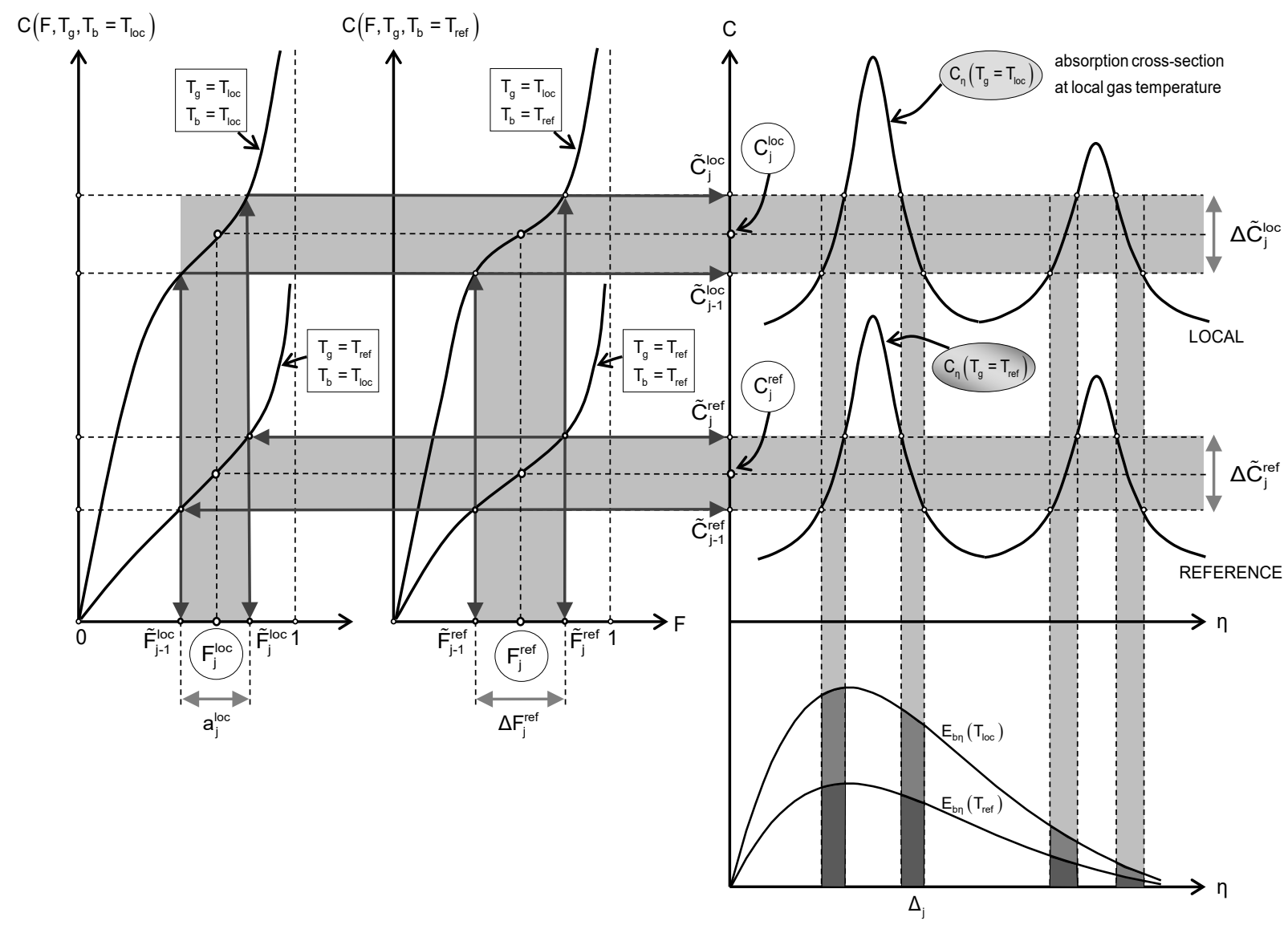

Figure 7. Visualization of the Generalized SLW reference approach based on the rank correlated spectrum assumption, which maintain fixed wavenumber intervals for spectral integration. 


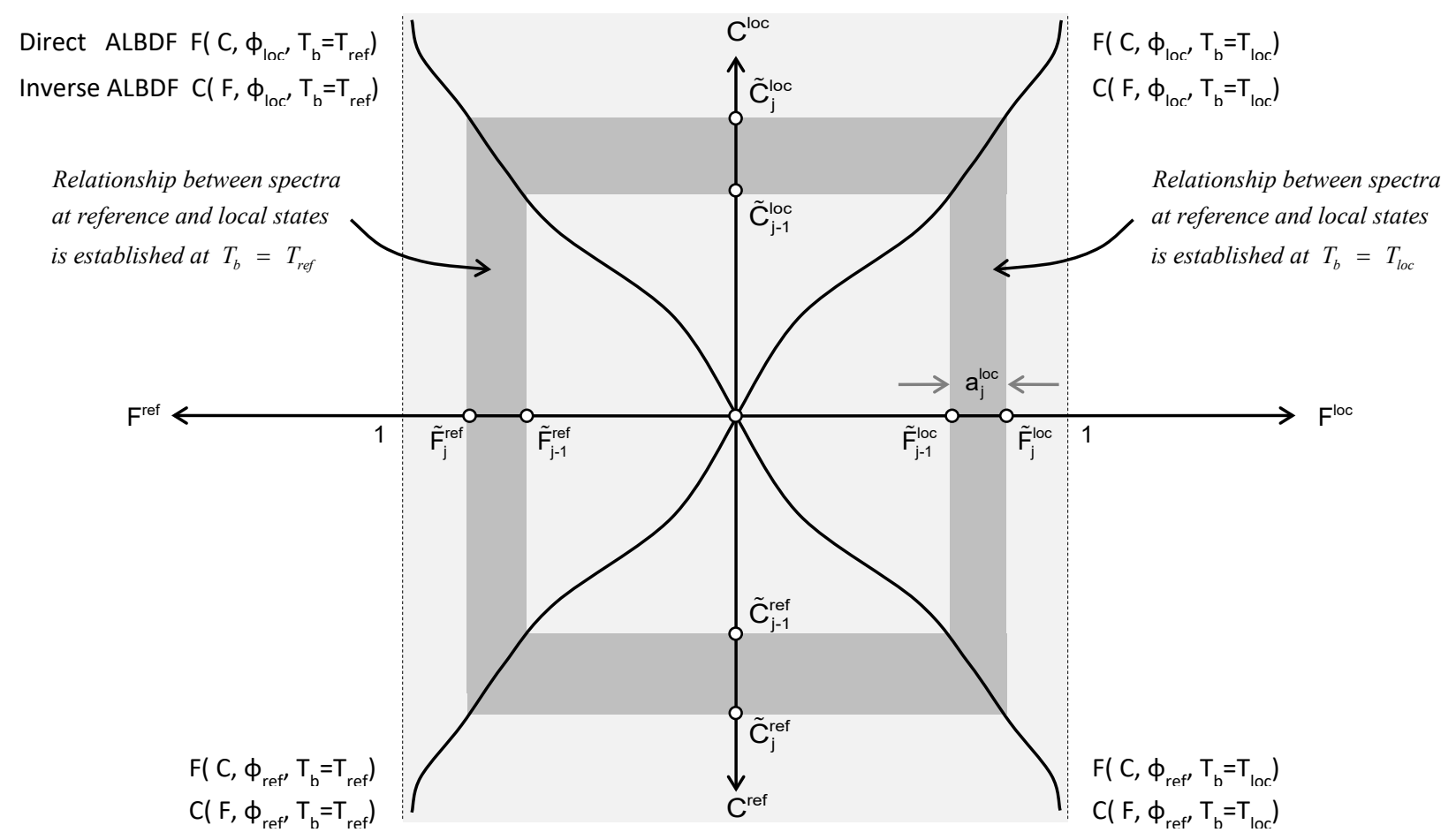

Figure 8. Visualization of all possible versions of the Generalized SLW reference approach. 


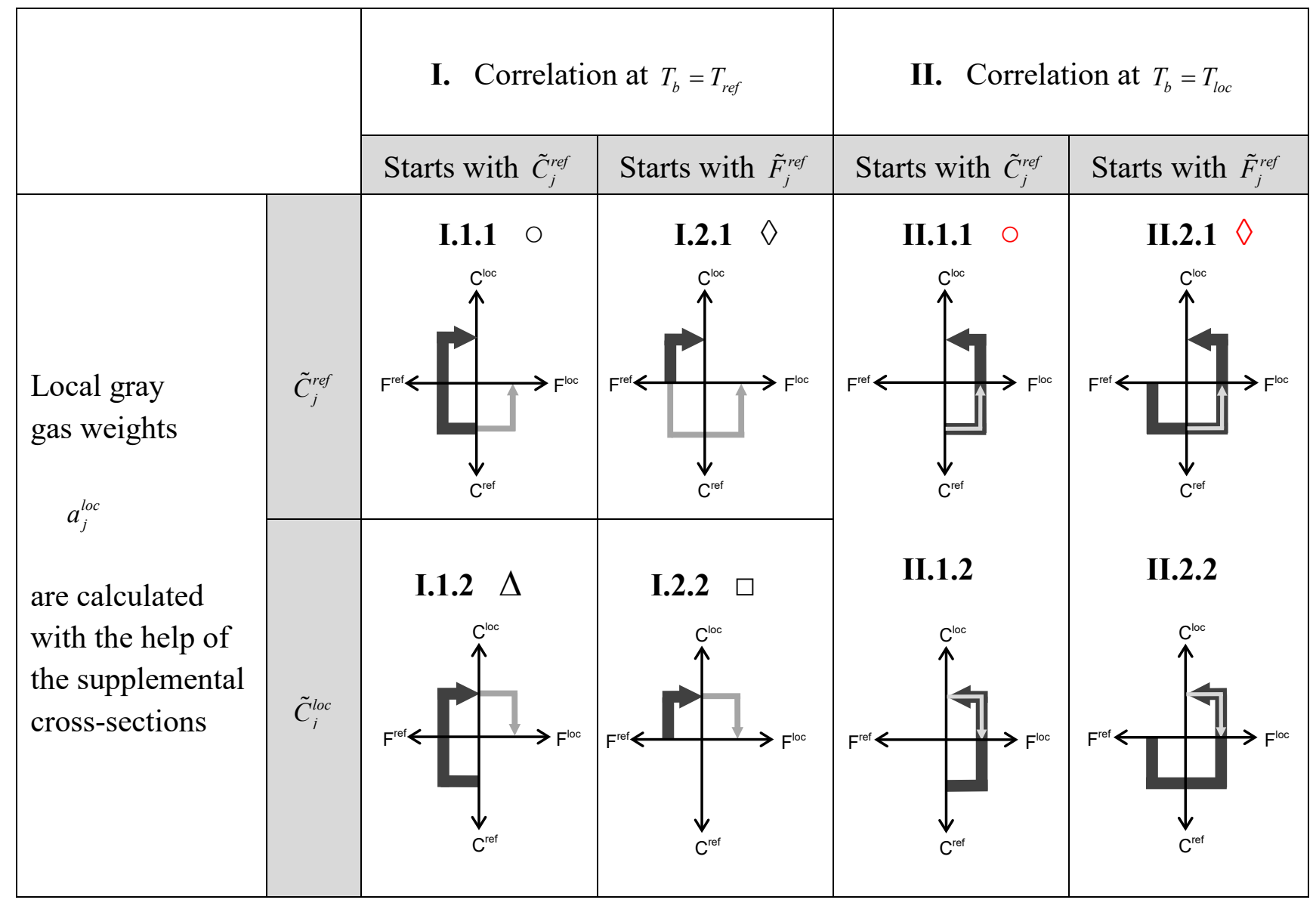

Figure 9. Schematic and classification of the different versions of the SLW reference approach: Arrows denote the method for: calculation of $\tilde{C}_{j}^{l o c}$ calculation of $a_{j}^{l o c}$. 


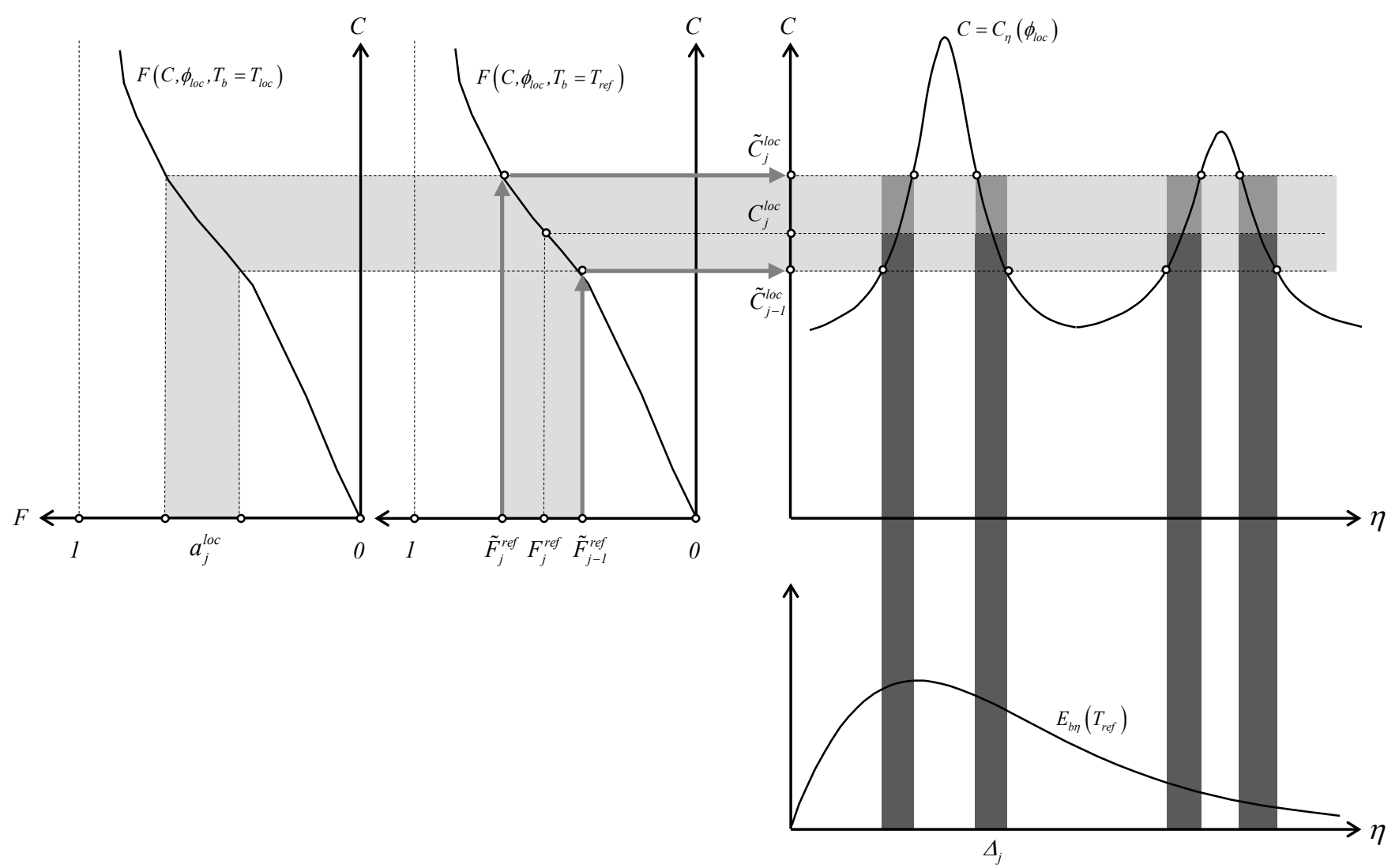

Figure 10. Graphical illustration of the Rank Correlated SLW spectral model (Method I.2.2). 

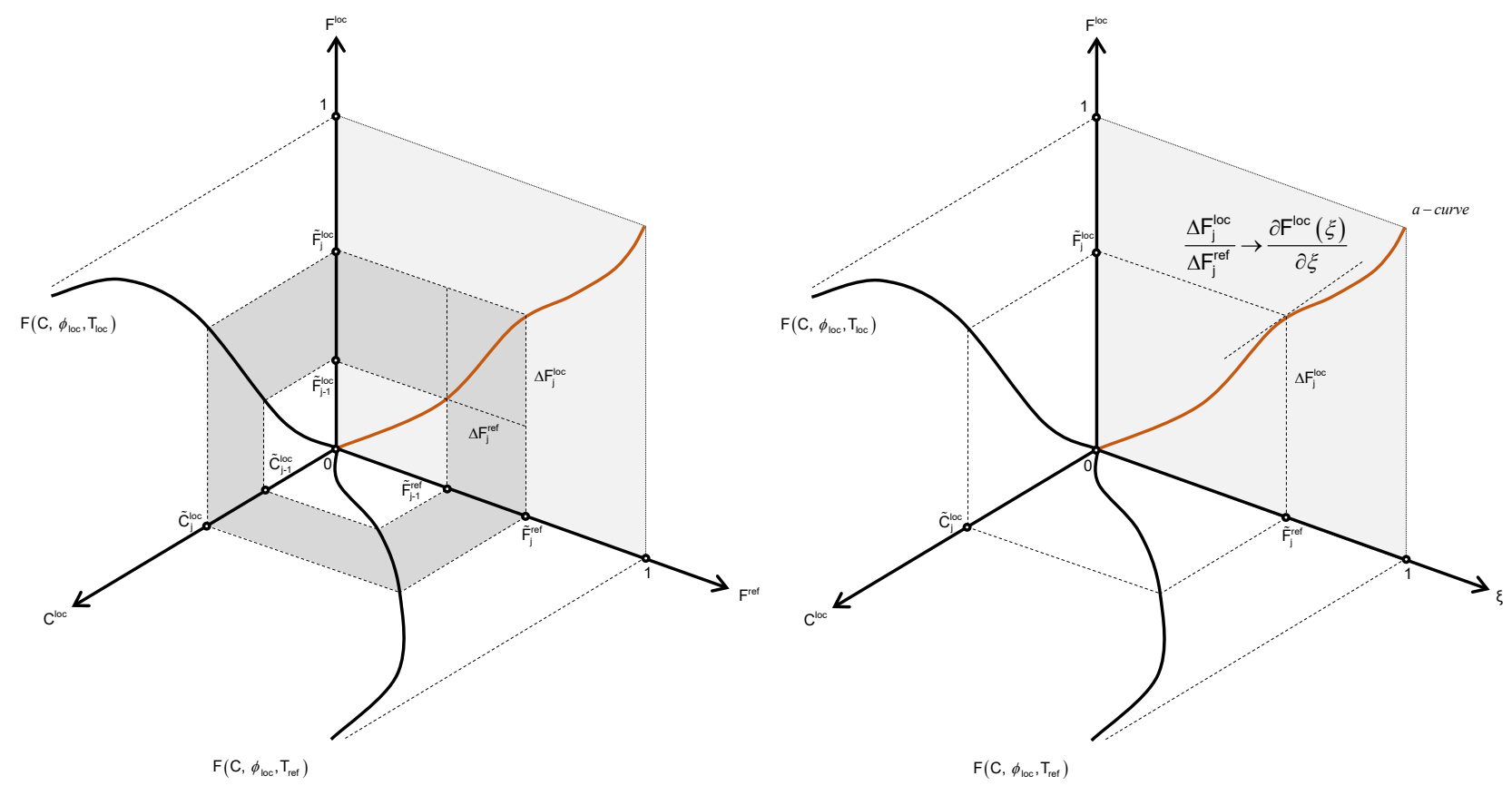

Figure 11. Finite increment form and continuous limit of the Rank Correlated SLW model (Method I.2.2). 

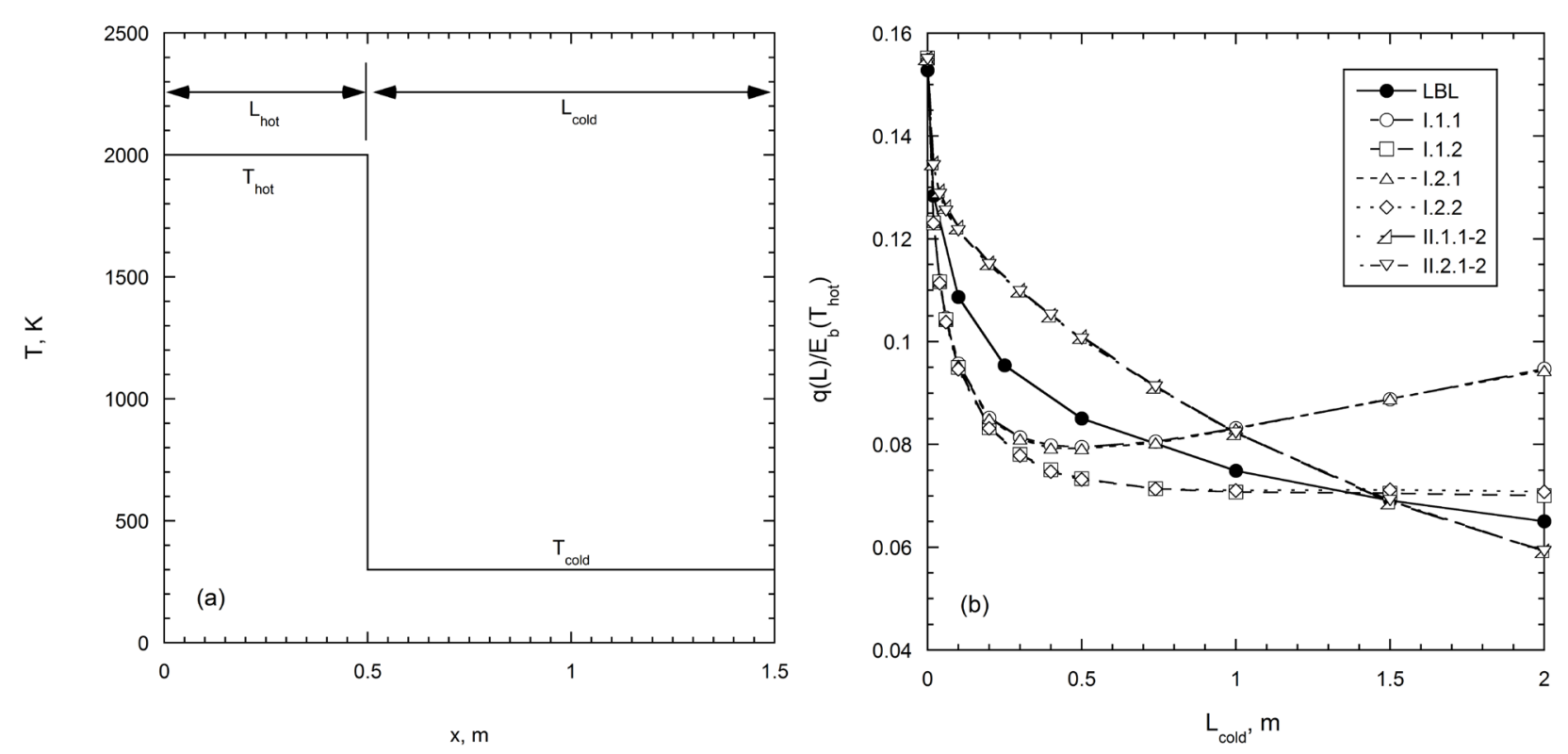

Figure 12. a) Temperature profile, and b) predicted normalized total wall radiative flux for Example 1. 

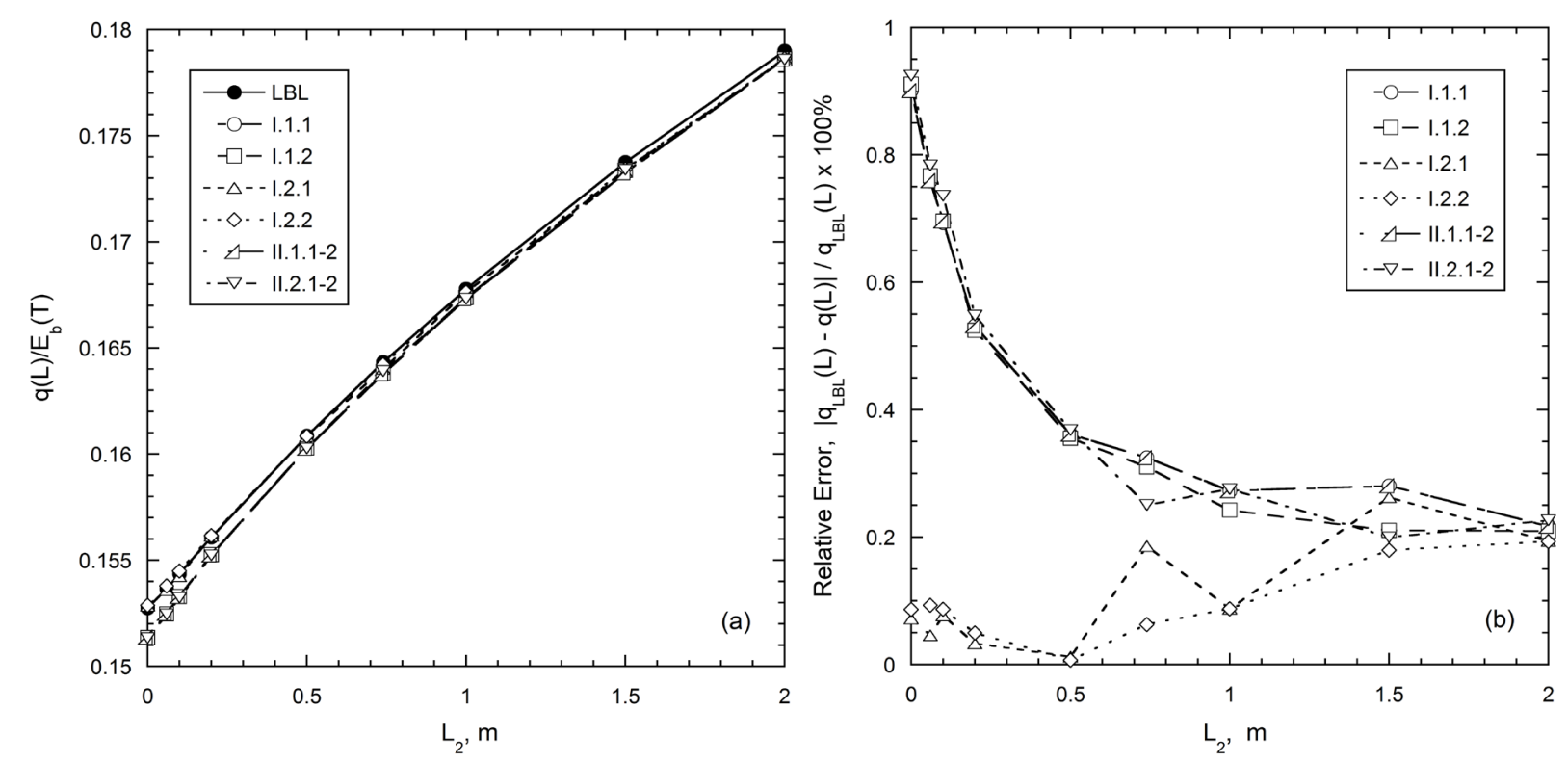

Figure 13. a) Normalized total wall radiative flux, and b) relative error of prediction of exiting flux, $\%$, for Example 2. 


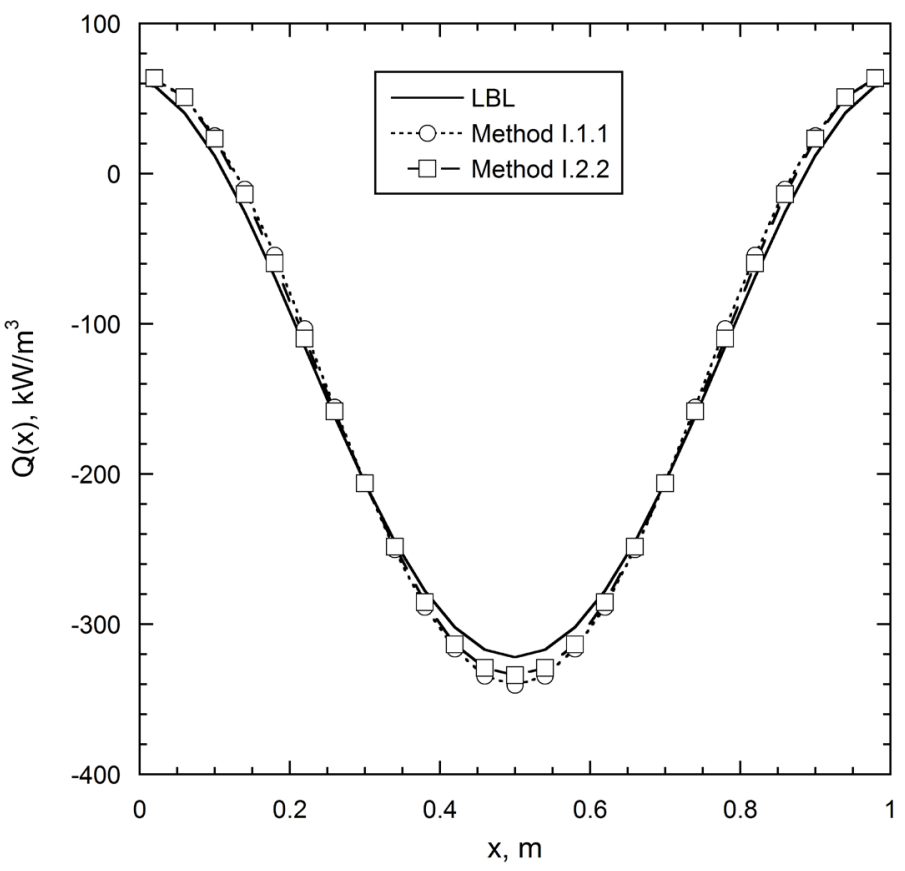

Figure 14. Predicted total divergence of the net radiative flux for Example 3. 

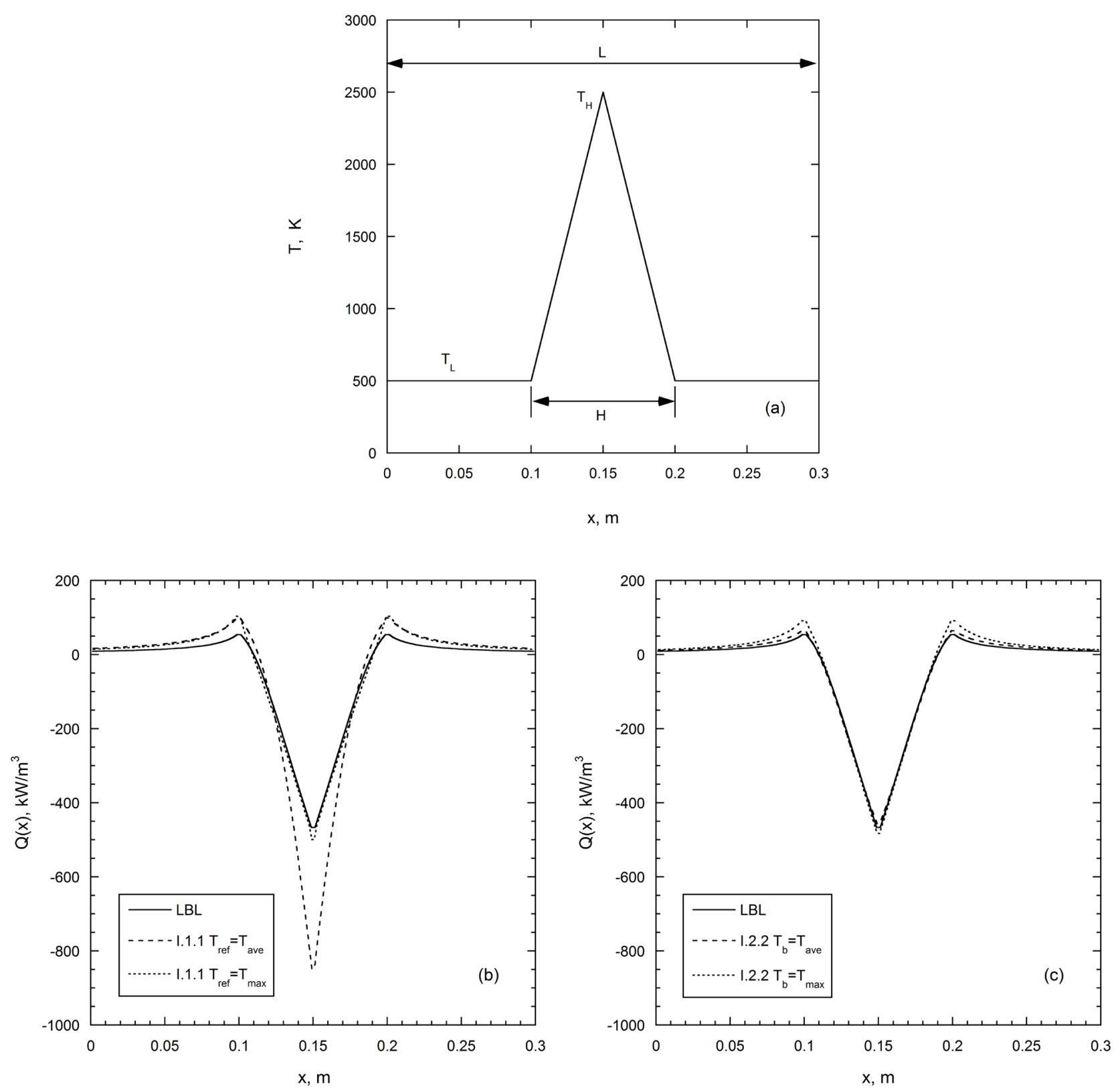

Figure 15. a) Gas temperature profile for Example 4, and predicted total divergence of the net radiative flux for b) Method I.1.1 (original SLW model reference method), and c) Method I.2.2 (Rank Correlated SLW model). 

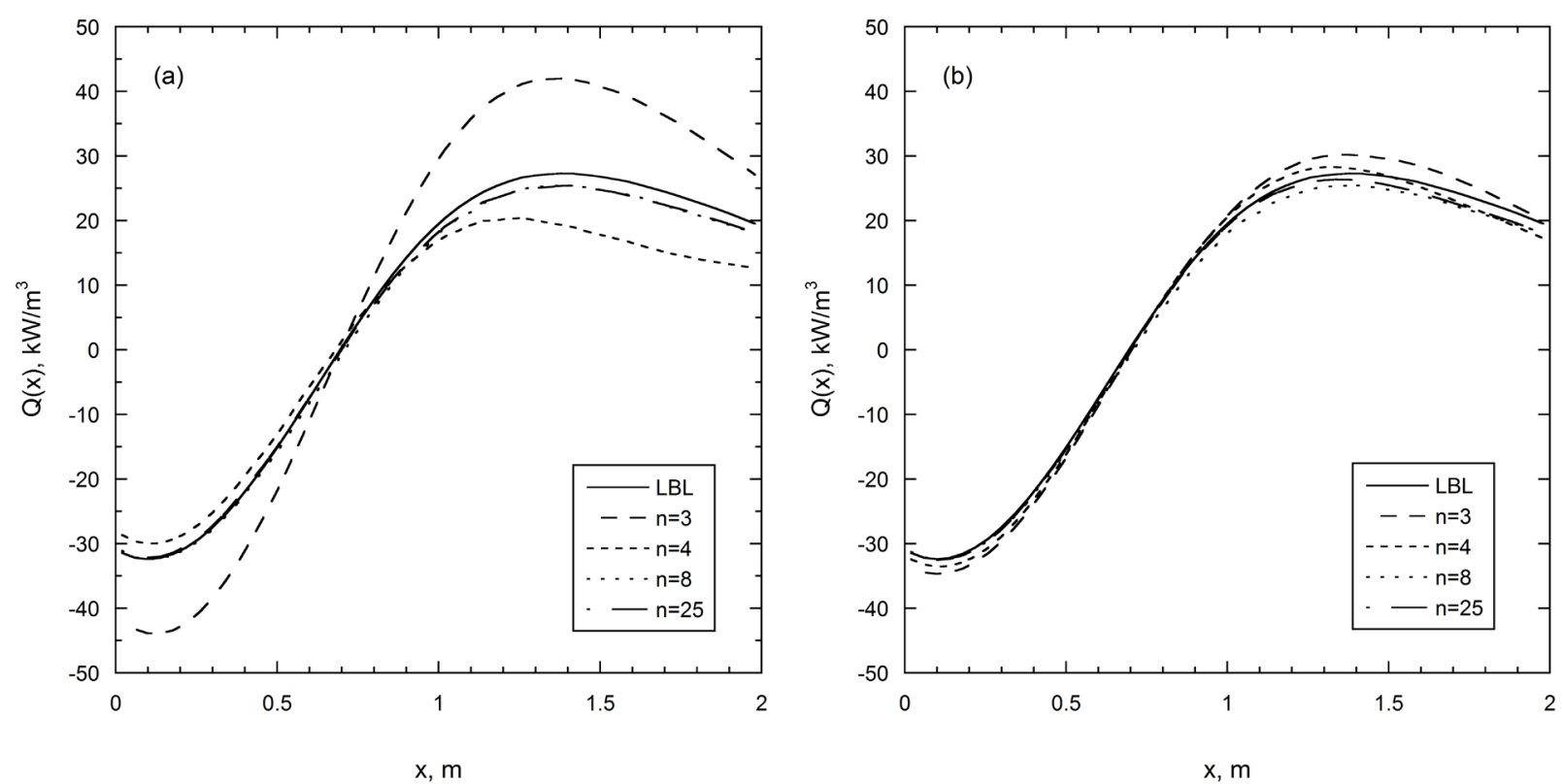

Figure 16. Predicted total divergence of the net radiative flux for Example 5: a) Method I.1.1 (original SLW reference method), and b) Method I.2.2 (Rank Correlated SLW model). 